\title{
GLOBAL WELL-POSEDNESS FOR DECONVOLUTION MAGNETOHYDRODYNAMICS MODELS WITH FRACTIONAL REGULARIZATION*
}

\author{
HANI ALI ${ }^{\dagger}$
}

\begin{abstract}
In this paper, we consider two Approximate Deconvolution Magnetohydrodynamics models which are related to Large Eddy Simulation. We first study existence and uniqueness of solutions in the double viscous case. Then, we study existence and uniqueness of solutions of the Approximate Deconvolution MHD model with magnetic diffusivity, but without kinematic viscosity. In each case, we give the optimal value of regularizations where we can prove global existence and uniqueness of the solutions. The second model includes the Approximate Deconvolution Euler Model as a particular case. Finally, an asymptotic stability result is shown in the double viscous case with weaker condition on the regularization parameter.
\end{abstract}

Key words. Magnetohydrodynamics, turbulence simulation and modeling, large-eddy simulations, partial differential equations.

AMS subject classifications. 76W05, 76B03, 76D05, 35Q30, 76F65, 76D03.

\section{Introduction.}

1.1. Mathematical setting of the problem. In this paper, we consider the following Approximate Deconvolution MHD equations in a three dimensional torus $\mathbb{T}_{3}$

$$
\left\{\begin{array}{l}
\partial_{t} \boldsymbol{w}+\nabla \cdot\left(\overline{D_{N, \theta}(\boldsymbol{w}) \otimes D_{N, \theta}(\boldsymbol{w})}\right)-\nabla \cdot(\overline{\boldsymbol{B} \otimes \boldsymbol{B}})-\nu \Delta \boldsymbol{w}+\nabla q=0, \\
\partial_{t} \boldsymbol{B}+\nabla \cdot\left(\boldsymbol{B} \otimes D_{N, \theta}(\boldsymbol{w})\right)-\nabla \cdot\left(D_{N, \theta}(\boldsymbol{w}) \otimes \boldsymbol{B}\right)-\mu \Delta \boldsymbol{B}=0 \\
\nabla \cdot \boldsymbol{w}=\nabla \cdot \boldsymbol{B}=0, \int_{\mathbb{T}_{3}} \boldsymbol{w}=\int_{\mathbb{T}_{3}} \boldsymbol{B}=0 \\
\boldsymbol{w}\left(t, \boldsymbol{x}+2 \pi \boldsymbol{e}_{\boldsymbol{j}}\right)=\boldsymbol{w}(t, \boldsymbol{x}), \boldsymbol{B}\left(t, \boldsymbol{x}+2 \pi \boldsymbol{e}_{\boldsymbol{j}}\right)=\boldsymbol{B}(t, \boldsymbol{x}), \boldsymbol{x} \in \mathbb{T}_{3}, t>0 \\
\boldsymbol{w}_{t=0}=\boldsymbol{w}_{0}=\overline{\boldsymbol{v}_{0}}, \boldsymbol{B}_{t=0}=\boldsymbol{B}_{0}
\end{array}\right.
$$

where the velocity field $\boldsymbol{w}(t, \boldsymbol{x})$, the magnetic field $\boldsymbol{B}(t, \boldsymbol{x})$, and the scalar pressure $p(t, \boldsymbol{x})$ are the unknowns, while $\nu \geq 0$ is the kinematic viscosity, $\mu \geq 0$ is the magnetic diffusion, and $D_{N, \theta}$ is a deconvolution operator given by

$$
D_{N, \theta}=\sum_{i=0}^{N}\left(I-\mathbb{A}_{\theta}^{-1}\right)^{i}, \quad \text { for } 0 \leq \theta \leq 1, \quad \text { and } N \geq 0
$$

Here

$$
\mathbb{A}_{\theta} \overline{\boldsymbol{v}}=\boldsymbol{v}:=\left(I+\alpha^{2 \theta}(-\Delta)^{\theta}\right) \overline{\boldsymbol{v}}
$$

denotes the Helmholtz operator with fractional regularization $\theta$ such that $\boldsymbol{v}$ represents the unfiltered velocity and $\overline{\boldsymbol{v}}$ is the filtered one. The parameter $\alpha>0$ is the length scale parameter that represents the width of the filter. In particular, in the filtered equations (1.1), the symbol "—" denotes the above Helmholtz filter (1.3), applied

\footnotetext{
* Received December 27, 2012; accepted for publication May 16, 2013.

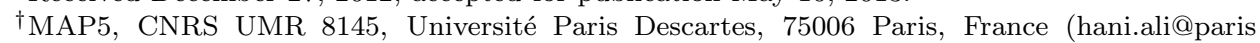
descartes.fr).
} 
component-by-component to the various tensor fields. If $\theta=0$, equations (1.1) become the incompressible MHD equations with initial data $\left(\boldsymbol{v}_{0}, \boldsymbol{B}_{0}\right)$. In the absence of the well-posedness theory of the MHD equations, the development of regularized equations is of major importance for both theoretical and practical purposes. Berselli et al. [4] have suggested two regularizations for the MHD equations. If we set $\theta=1$ in (1.1), we recover one of the models studied in [4]. For more details about the deconvolution models and the related background, see $[15,16,8]$.

The goal of this paper is to establish the existence and the uniqueness of a solution to the system (1.1) with fractional regularization $\theta$. First, we will consider the double viscous case of $(1.1)(\nu>0, \mu>0)$ in section 3 and then the inviscid case $(\nu=0$, $\mu>0$ ) in section 4 . In both cases, we will look for the optimal values of $\theta$ where we can prove global existence and uniqueness of the solutions for any fixed $N$.

Before specifying what we mean by a solution to (1.1) in each of these two cases and giving our main results, we introduce suitable function spaces.

1.2. Functional spaces. We denote by $L^{p}\left(\mathbb{T}_{3}\right)$ and $H^{s}\left(\mathbb{T}_{3}\right),(s \geq-1,1 \leq p \leq$ $\infty$,) the usual Lebesgue and Sobolev spaces over $\mathbb{T}_{3}$, and define the Bochner spaces $C(0, T ; X)$, and $L^{p}(0, T ; X)$ in the standard way. The Sobolev spaces $\boldsymbol{H}^{s}=H^{s}\left(\mathbb{T}_{3}\right)^{3}$, of mean-free functions are classically characterized in terms of the Fourier series as follows,

$$
\boldsymbol{H}^{s}=\left\{\boldsymbol{v}(\boldsymbol{x})=\sum_{\boldsymbol{k} \in \mathbb{Z}^{3} \backslash\{0\}} \boldsymbol{c}_{\boldsymbol{k}} e^{i \boldsymbol{k} \cdot \boldsymbol{x}},\left(\boldsymbol{c}_{\boldsymbol{k}}\right)^{*}=\boldsymbol{c}_{-\boldsymbol{k}}, \boldsymbol{c}_{0}=0,\|\boldsymbol{v}\|_{s, 2}^{2}=\sum_{\boldsymbol{k} \in \mathbb{Z}^{3} \backslash\{0\}}|\boldsymbol{k}|^{2 s}\left|\boldsymbol{c}_{\boldsymbol{k}}\right|^{2}<\infty\right\},
$$

where $\left(\boldsymbol{c}_{\boldsymbol{k}}\right)^{*}$ denotes the complex conjugate $\boldsymbol{c}_{\boldsymbol{k}}$.

In addition, we introduce

$$
\begin{aligned}
\boldsymbol{H}_{\sigma}^{s} & =\left\{\boldsymbol{v} \in \boldsymbol{H}^{s} ; \nabla \cdot \boldsymbol{v}=0 \text { in } \mathbb{T}_{3}\right\} \\
\boldsymbol{H}^{-s} & =\left(\boldsymbol{H}^{s}\right)^{\prime}, \quad \boldsymbol{L}^{2}=\boldsymbol{H}^{0}, \quad \boldsymbol{L}_{\sigma}^{2}=\boldsymbol{H}_{\sigma}^{0}
\end{aligned}
$$

1.3. Main results. In this subsection, we define the notion of a solution to the system (1.1) and we give precisely the main results of this paper.

1.3.1. Definitions of regular weak solutions. We first consider the double viscous case of $(1.1)(\nu>0, \mu>0)$.

Definition 1.1. Let $\nu>0, \mu>0, \boldsymbol{v}_{0} \in \boldsymbol{L}_{\sigma}^{2}$ and $\boldsymbol{B}_{0} \in \boldsymbol{L}_{\sigma}^{2}$. For any $0 \leq \theta \leq 1$ and $0 \leq N<\infty$ we say that the triplet $(\boldsymbol{w}, \boldsymbol{B}, q)$ is a "regular" weak solution to (1.1) if for any $T>0$ the following properties are satisfied:

$$
\begin{aligned}
\boldsymbol{w} & \in \mathcal{C}\left(0, T ; \boldsymbol{H}_{\sigma}^{\theta}\right) \cap L^{2}\left(0, T ; \boldsymbol{H}_{\sigma}^{1+\theta}\right), \quad \boldsymbol{B} \in \mathcal{C}\left(0, T ; \boldsymbol{L}_{\sigma}^{2}\right) \cap L^{2}\left(0, T ; \boldsymbol{H}_{\sigma}^{1}\right), \\
\partial_{t} \boldsymbol{w} & \in L^{2}\left(0, T ; \boldsymbol{H}^{2 \theta-\frac{3}{2}}\right), \quad \partial_{t} \boldsymbol{B} \in L^{2}\left(0, T ; \boldsymbol{H}^{-1}\right), \\
q & \in L^{2}\left(0, T ; H^{2 \theta-\frac{1}{2}}\left(\mathbb{T}_{3}\right)\right),
\end{aligned}
$$

the triplet $(\boldsymbol{w}, \boldsymbol{B}, q)$ fulfill

$$
\begin{gathered}
\int_{0}^{T}\left\langle\partial_{t} \boldsymbol{w}, \boldsymbol{\varphi}\right\rangle-\left\langle\overline{D_{N, \theta}(\boldsymbol{w}) \otimes D_{N, \theta}(\boldsymbol{w})}, \nabla \boldsymbol{\varphi}\right\rangle+\nu\langle\nabla \boldsymbol{w}, \nabla \boldsymbol{\varphi}\rangle+\langle\nabla q, \boldsymbol{\varphi}\rangle d t \\
\left.+\int_{0}^{T} \overline{\langle(\boldsymbol{B}) \otimes(\boldsymbol{B})}, \nabla \boldsymbol{\varphi}\right\rangle d t=0 \quad \text { for all } \boldsymbol{\varphi} \in L^{2}\left(0, T ; \boldsymbol{H}^{\frac{3}{2}-2 \theta}\right),
\end{gathered}
$$


DECONVOLUTION MHD WITH FRACTIONAL REGULARIZATION

$$
\begin{aligned}
& \int_{0}^{T}\left\langle\partial_{t} \boldsymbol{B}, \boldsymbol{\varphi}\right\rangle+\left\langle D_{N, \theta}(\boldsymbol{w}) \otimes \boldsymbol{B}, \nabla \boldsymbol{\varphi}\right\rangle+\mu\langle\nabla \boldsymbol{B}, \nabla \boldsymbol{\varphi}\rangle d t \\
& \quad-\int_{0}^{T}\left\langle\boldsymbol{B} \otimes D_{N, \theta}(\boldsymbol{w}), \nabla \boldsymbol{\varphi}\right\rangle d t=0 \quad \text { for all } \boldsymbol{\psi} \in L^{2}\left(0, T ; \boldsymbol{H}^{1}\right) .
\end{aligned}
$$

Moreover,

$$
\boldsymbol{w}(0)=\boldsymbol{w}_{0} \quad \text { and } \quad \boldsymbol{B}(0)=\boldsymbol{B}_{0}
$$

Then, we give the definition of the solution of (1.1) with partial viscous term $(\nu=0, \mu>0)$.

Definition 1.2. Let $\nu=0, \mu>0, \boldsymbol{v}_{0} \in \boldsymbol{L}_{\sigma}^{2}$ and $\boldsymbol{B}_{0} \in \boldsymbol{L}_{\sigma}^{2}$. For any $0 \leq \theta \leq 1$ and $0 \leq N<\infty$ we say that the triplet $(\boldsymbol{w}, \boldsymbol{B}, q)$ is a weak solution to (1.1) if for any $T>0$ the following properties are satisfied:

$$
\begin{aligned}
\boldsymbol{w} & \in \mathcal{C}\left(0, T ; \boldsymbol{H}_{\sigma}^{\theta}\right), \\
\boldsymbol{B} & \in \mathcal{C}\left(0, T ; \boldsymbol{L}_{\sigma}^{2}\right) \cap L^{2}\left(0, T ; \boldsymbol{H}_{\sigma}^{1}\right), \\
\partial_{t} \boldsymbol{w} & \in L^{4}\left(0, T ; \boldsymbol{H}^{2 \theta-2}\right) \cap L^{\frac{6}{5}}\left(0, T ; \boldsymbol{H}^{2 \theta-\frac{5}{6}}\right), \\
\partial_{t} \boldsymbol{B} & \in L^{2}\left(0, T ; \boldsymbol{H}^{-1}\right), \\
q & \in L^{2}\left(0, T ; H^{2 \theta-1}\left(\mathbb{T}_{3}\right)\right),
\end{aligned}
$$

the triplet $(\boldsymbol{w}, \boldsymbol{B}, q)$ fulfill

$$
\begin{array}{r}
\int_{0}^{T}\left\langle\partial_{t} \boldsymbol{w}, \boldsymbol{\varphi}\right\rangle-\left\langle\overline{D_{N, \theta}(\boldsymbol{w}) \otimes D_{N, \theta}(\boldsymbol{w})}, \nabla \boldsymbol{\varphi}\right\rangle+\langle\nabla q, \boldsymbol{\varphi}\rangle d t \\
\left.+\int_{0}^{T} \overline{\langle(\boldsymbol{B}) \otimes(\boldsymbol{B})}, \nabla \boldsymbol{\varphi}\right\rangle d t=0 \quad \text { for all } \boldsymbol{\varphi} \in L^{\frac{4}{3}}\left(0, T ; \boldsymbol{H}^{2-2 \theta}\right), \\
\int_{0}^{T}\left\langle\partial_{t} \boldsymbol{B}, \boldsymbol{\varphi}\right\rangle+\left\langle D_{N, \theta}(\boldsymbol{w}) \otimes \boldsymbol{B}, \nabla \boldsymbol{\varphi}\right\rangle+\mu\langle\nabla \boldsymbol{B}, \nabla \boldsymbol{\varphi}\rangle d t \\
-\int_{0}^{T}\left\langle\boldsymbol{B} \otimes D_{N, \theta}(\boldsymbol{w}), \nabla \boldsymbol{\varphi}\right\rangle d t=0 \quad \text { for all } \boldsymbol{\psi} \in L^{2}\left(0, T ; \boldsymbol{H}^{1}\right) .
\end{array}
$$

Moreover,

$$
\boldsymbol{w}(0)=\boldsymbol{w}_{0} \quad \text { and } \quad \boldsymbol{B}(0)=\boldsymbol{B}_{0}
$$

Furthermore, if, $\boldsymbol{B}_{0} \in \boldsymbol{H}_{\sigma}^{1}$ we say that $(\boldsymbol{w}, \boldsymbol{B}, q)$ is a "regular" weak solution to (1.1) if it is a weak solution, and in addition $\boldsymbol{w}$ and $\boldsymbol{B}$ satisfy

$$
\begin{aligned}
& \boldsymbol{w} \in L^{\infty}\left(0, T ; \boldsymbol{H}_{\sigma}^{2 \theta}\right) \\
& \boldsymbol{B} \in \mathcal{C}\left(0, T ; \boldsymbol{H}_{\sigma}^{1}\right) \cap L^{2}\left(0, T ; \boldsymbol{H}_{\sigma}^{2}\right)
\end{aligned}
$$

Note that the term "regular" in the above definitions is used when weak solutions are unique and do not develop a finite-time singularities. 
1.3.2. Statement of the main results. The main results of this paper are the following:

TheOREm 1.1. Consider the Approximate Deconvolution MHD equations (1.1) with $\nu>0$ and $\mu>0$. Assume $\boldsymbol{v}_{0} \in L_{\sigma}^{2}$ and $\boldsymbol{B}_{0} \in \boldsymbol{L}_{\sigma}^{2}$. Let $\theta \geq \frac{1}{2}$ and let $0 \leq N<\infty$ is given and fixed. Then (1.1) with initial data $\left(\boldsymbol{v}_{0}, \boldsymbol{B}_{0}\right)$ has a unique regular weak solution.

We note that the value $\theta=\frac{1}{2}$ is optimal in order to get existence and uniqueness of weak solutions with $\boldsymbol{L}_{\sigma}^{2}$-initial data in the energy spaces, i.e. $\mathcal{C}\left(0, T ; \boldsymbol{H}_{\sigma}^{\theta}\right) \cap$ $L^{2}\left(0, T ; \boldsymbol{H}_{\sigma}^{1+\theta}\right)$, and $\mathcal{C}\left(0, T ; \boldsymbol{L}_{\sigma}^{2}\right) \cap L^{2}\left(0, T ; \boldsymbol{H}_{\sigma}^{1}\right)$. The value $\theta=\frac{1}{2}$ can be slightly relaxed by using more delicate tools as in $[17,20]$. However, in this case taking the initial data only in $\boldsymbol{L}_{\sigma}^{2}$ is not allowed. More details on this topic will be given in a forthcoming paper.

Theorem 1.2. Consider the Approximate Deconvolution MHD equations (1.1) with $\nu=0$ and $\mu>0$. Assume $\boldsymbol{v}_{0} \in L_{\sigma}^{2}$ and $\boldsymbol{B}_{0} \in L_{\sigma}^{2}$. Let $\theta \geq \frac{5}{6}$ and let $0 \leq$ $N<\infty$ is given and fixed. Then (1.1) with initial data $\left(\boldsymbol{v}_{0}, \boldsymbol{B}_{0}\right)$ has a weak solution. Furthermore, if $\boldsymbol{B}_{0} \in \boldsymbol{H}_{\sigma}^{1}$ then (1.1) with initial data $\left(\boldsymbol{v}_{0}, \boldsymbol{B}_{0}\right)$ has a unique "regular" weak solution among the class of weak solutions.

Theorem 1.2 includes a special case that deserves a separate formulation. If we consider the case $\boldsymbol{B}=0$, then equations (1.1) with $\nu=0$ become the Approximate Deconvolution Model of Euler equations (ADE) with periodic boundary conditions. Consequently, Theorem 1.2 reduces to the following statement.

Corollary 1.1. Assume $\boldsymbol{v}_{0} \in L_{\sigma}^{2}$. Let $\theta \geq \frac{5}{6}$ and $0 \leq N<\infty$ given and fixed numbers. Then the Approximate Deconvolution Euler equations (ADE) with initial data $\boldsymbol{v}_{0}$ have a unique regular weak solution $\boldsymbol{w} \in \mathcal{C}\left(0, T ; \boldsymbol{H}_{\sigma}^{\theta}\right)$. This regular weak solution satisfies the energy equality:

$$
\frac{1}{2}\left\|\mathbb{A}_{\theta}^{\frac{1}{2}} D_{N, \theta}^{\frac{1}{2}}(\boldsymbol{w})(t)\right\|_{2}^{2}=\frac{1}{2}\left\|\mathbb{A}_{\theta}^{\frac{1}{2}} D_{N, \theta}^{\frac{1}{2}}\left(\overline{\boldsymbol{v}}_{0}\right)\right\|_{2}^{2} .
$$

REMARK 1.1. From the above energy equality, we can deduce by using the properties of $D_{N, \theta}$ and $\mathbb{A}_{\theta}$ (see section 2.1), the following inequality,

$$
\|\boldsymbol{w}(t)\|_{2}^{2}+\alpha^{2 \theta}\|\boldsymbol{w}(t)\|_{\theta, 2}^{2} \leq\left\|\boldsymbol{v}_{0}\right\|_{2}^{2}
$$

Using the same techniques as in [11], we can derive from (1.21) the following criterion for finite-time blow-up of the Euler equations.

Blow-up criterion: Assume $\boldsymbol{v}_{0} \in \boldsymbol{H}_{\sigma}^{s}$, for some $s>3$. Suppose that there exists a finite time $T^{*}>0$ such that the solution $\boldsymbol{w}$ of (ADE) with initial data $\boldsymbol{v}_{0}$ satisfies the below inequality for each $\alpha>0$ and $0 \leq N<\infty$ :

$$
\sup _{t \in\left[0, T^{*}\right]} \limsup _{\alpha \rightarrow 0} \alpha^{2 \theta}\|\boldsymbol{w}(t)\|_{\theta, 2}^{2}>0 .
$$

Then the Euler equations with initial data $\boldsymbol{v}_{0}$ develop a singularity in the interval $\left[0, T^{*}\right]$.

Let us briefly review some existence results for regularizations of the magnetohydrodynamics equations; we will not attempt to address exhaustive references in this paper. 
The existence and uniqueness of the inviscid Voigt regularization of the MHD equations are studied in $[12,6]$. The existence and uniqueness of a related regularization for the MHD equations, LES for MHD, are studied in [2]. When $\theta=1$ and $N$ fixed, the global well-posedness of the double viscous problem has been proven in [4]. When $\boldsymbol{B}=0$ and $\theta=1$, equations (1.1) become the well-known Approximate Deconvolution Model (ADM). Recently, the authors [5] have proven that the ADM with fractional regularization has a unique regular weak solution when $\theta>\frac{3}{4}$. The question of the limit behavior of the ADM solutions when $N$ tends to infinity is studied also in [4] when $\theta>\frac{3}{4}$. The above uniqueness and convergence results are improved in [3] to cover the range when $\theta \geq \frac{1}{6}$. The deconvolution operator, for different values of $\theta$, was used in [9] to study the rate of convergence of the ADM model to the mean Navier-Stokes Equations. Very recently, Berselli et al. [4] have adapted the results in [5] to the Deconvolution MHD equations (1.1).

In this paper, our approach is partially inspired from the recent studies of Berselli et al. $[4,5]$. We point out that in [4] the authors consider only the double viscous case with $\theta=1$. In this paper, we study not only the double viscous case but also the partial inviscid case with fractional regularization. In the double viscous case, we derive some apriori estimates that are uniform with respect to $N$. These uniform estimates combined with a compactness argument allow us to study the limit behavior of the solutions of the Deconvolution MHD model when $N$ tends to infinity, for $\theta \geq \frac{1}{2}$. Unfortunately, in the partial viscous case, we are not able to use the method developed in this paper to study the limit behavior of the solutions when $N$ tends to infinity. This question remains open.

The remaining part of the paper is organized as follows. In section 2, we start by giving some preliminaries about the deconvolution operator $D_{N, \theta}$ and the product of two functions in Sobolev spaces. Section 3 provides existence and uniqueness results in the double viscous case where $0 \leq N<\infty$ is fixed and $\theta \geq \frac{1}{2}$. Section 4 provides existence and uniqueness results in the partial inviscid case where $0 \leq N<\infty$ is fixed and $\theta \geq \frac{5}{6}$. The last section provides a result of consistency and stability which illustrates how the system approximates the MHD equations.

\section{Preliminaries and auxiliary results.}

2.1. The deconvolution operator. In this paper we consider a generalized deconvolution operator $[5,1]$. This deconvolution operator is constructed by using $\mathbb{A}_{\theta}$, the Helmholtz equation with fractional regularization.

For $p=-1,-\frac{1}{2}, \frac{1}{2}$ or 1 , the pseudo-differential operator $\mathbb{A}_{\theta}^{p}$ is defined in the periodic case as

$$
\mathbb{A}_{\theta}^{p}\left(\sum_{\boldsymbol{k} \in \mathbb{Z}^{3} \backslash\{0\}} \boldsymbol{c}_{\boldsymbol{k}} e^{\mathrm{i} \mathbf{k} \cdot \mathbf{x}}\right)=\sum_{\boldsymbol{k} \in \mathbb{Z}^{3} \backslash\{0\}}\left(1+\alpha^{2 \theta}|\boldsymbol{k}|^{2 \theta}\right)^{p} \boldsymbol{c}_{\boldsymbol{k}} e^{\mathrm{i} \mathbf{k} \cdot \mathbf{x}}, \text { for any } 0 \leq \theta \leq 1,
$$

and in particular $\mathbb{A}_{\theta}^{-1}$ verifies

$$
\left\|\mathbb{A}_{\theta}^{-1}(\boldsymbol{v})\right\|_{s+2 \theta, 2} \leq \alpha^{-2 \theta}\|\boldsymbol{v}\|_{s, 2} .
$$

This operator commutes with differentiation under periodic boundary conditions. In the analysis we will need the following relations which follow from (2.1)

$$
\left(\mathbb{A}_{\theta}^{-\frac{1}{2}}(\boldsymbol{v}), \mathbb{A}_{\theta}^{\frac{1}{2}}(\boldsymbol{w})\right)=(\boldsymbol{v}, \boldsymbol{w}), \text { for any } \boldsymbol{v} \in \boldsymbol{L}^{2} \text { and } \boldsymbol{w} \in \boldsymbol{H}^{\theta},
$$




$$
\left\|\mathbb{A}_{\theta}(\boldsymbol{v})\right\|_{2}^{2}=\|\boldsymbol{v}\|_{2}^{2}+2 \alpha^{2 \theta}\|\boldsymbol{v}\|_{\theta, 2}^{2}+\alpha^{4 \theta}\|\boldsymbol{v}\|_{2 \theta, 2}^{2}, \text { for any } \boldsymbol{v} \in \boldsymbol{H}^{2 \theta} .
$$

The pseudo-differential operator $D_{N, \theta}$ is given by

$$
\begin{aligned}
& D_{N, \theta}\left(\sum_{\boldsymbol{k} \in \mathbb{Z}^{3} \backslash\{0\}} \boldsymbol{c}_{\boldsymbol{k}} e^{\mathrm{i} \mathbf{k} \cdot \mathbf{x}}\right) \\
& =\sum_{\boldsymbol{k} \in \mathbb{Z}^{3} \backslash\{0\}}\left(1+\alpha^{2 \theta}|\boldsymbol{k}|^{2 \theta}\right)\left(1-\left(\frac{\alpha^{2 \theta}|\boldsymbol{k}|^{2 \theta}}{1+\alpha^{2 \theta}|\boldsymbol{k}|^{2 \theta}}\right)^{N+1}\right) \boldsymbol{c}_{\boldsymbol{k}} e^{\mathrm{i} \mathbf{k} \cdot \mathbf{x}} .
\end{aligned}
$$

Thus,

$$
D_{N, \theta}\left(\sum_{\boldsymbol{k} \in \mathbb{Z}^{3} \backslash\{0\}} \boldsymbol{c}_{\boldsymbol{k}} e^{\mathrm{i} \mathbf{k} \cdot \mathbf{x}}\right)=\sum_{\boldsymbol{k} \in \mathbb{Z}^{3} \backslash\{0\}} \widehat{D_{N, \theta}}(\boldsymbol{k}) \boldsymbol{c}_{\boldsymbol{k}} e^{\mathrm{i} \mathbf{k} \cdot \mathbf{x}},
$$

where we have for all $\boldsymbol{k} \in \mathbb{Z}^{3} \backslash\{0\}$,

$$
\begin{aligned}
\widehat{D_{0, \theta}}(\boldsymbol{k}) & =1 \\
1 \leq \widehat{D_{N, \theta}}(\boldsymbol{k}) & \leq N+1 \quad \text { for each } N>0 \\
\text { and } \widehat{D_{N, \theta}}(\boldsymbol{k}) & \leq \widehat{\mathbb{A}_{\theta}}:=\left(1+\alpha^{2 \theta}|\boldsymbol{k}|^{2 \theta}\right) \text { for a fixed } \alpha>0
\end{aligned}
$$

The following elementary lemma is given in [5] and will play an important role.

Lemma 2.1. For all $s \geq-1, \boldsymbol{k} \in \mathbb{Z}^{3} \backslash\{0\}$, and for each $N>0$ there exist a constant $C>0$ such that for all $\boldsymbol{v}$ sufficiently smooth we have

$$
\begin{aligned}
& \|\boldsymbol{v}\|_{s, 2} \leq\left\|D_{N, \theta}(\boldsymbol{v})\right\|_{s, 2} \leq(N+1)\|\boldsymbol{v}\|_{s, 2}, \\
& \|\boldsymbol{v}\|_{s, 2} \leq C\left\|D_{N, \theta}(\boldsymbol{v})\right\|_{s, 2} \leq C\left\|\mathbb{A}_{\theta}^{\frac{1}{2}} D_{N, \theta}^{\frac{1}{2}}(\boldsymbol{v})\right\|_{s, 2}, \\
& \left\|\mathbb{A}_{\theta}^{\frac{1}{2}} D_{N, \theta}^{\frac{1}{2}}(\overline{\boldsymbol{v}})\right\|_{s, 2} \leq\|\boldsymbol{v}\|_{s, 2} \\
& \|\boldsymbol{v}\|_{s+\theta, 2} \leq C(\alpha)\left\|\mathbb{A}_{\theta}^{\frac{1}{2}} D_{N, \theta}^{\frac{1}{2}}(\boldsymbol{v})\right\|_{s, 2} .
\end{aligned}
$$

2.2. Product laws in Sobolev spaces. In this subsection, we recall a known result concerning the product of two functions in Sobolev spaces (see [7] for instance).

Lemma 2.2. Let $\boldsymbol{v} \in \boldsymbol{H}^{s_{1}}, \boldsymbol{w} \in \boldsymbol{H}^{s_{2}}$ with $s_{1}+s_{2}>0$ and $s_{1}, s_{2}<\frac{3}{2}$. Then $\boldsymbol{v} \otimes \boldsymbol{w} \in \boldsymbol{H}^{s_{1}+s_{2}-\frac{3}{2}}$ and there exists a constant $C>0$ such that

$$
\|\boldsymbol{v} \otimes \boldsymbol{w}\|_{s_{1}+s_{2}-\frac{3}{2}, 2} \leq C\|\boldsymbol{v}\|_{s_{1}, 2}\|\boldsymbol{w}\|_{s_{2}, 2} .
$$

The following lemma will play an important role in the proof of the main results.

Lemma 2.3. (i) Let $0<\theta<1$. If $\boldsymbol{v} \in L^{\infty}\left(0, T ; \boldsymbol{H}^{\theta}\right) \cap L^{2}\left(0, T ; \boldsymbol{H}^{1+\theta}\right)$, then $\boldsymbol{v} \otimes \boldsymbol{v} \in L^{2}\left(0, T ; H^{2 \theta-\frac{1}{2}}\left(\mathbb{T}_{3}\right)^{3 \times 3}\right)$ and there exists a constant $C>0$ such that $\|\boldsymbol{v} \otimes \boldsymbol{v}\|_{L^{2}\left(0, T ; H^{2 \theta-\frac{1}{2}}\left(\mathbb{T}_{3}\right)^{3 \times 3}\right)} \leq C\|\boldsymbol{v}\|_{L^{4}\left(0, T ; \boldsymbol{H}^{\theta+\frac{1}{2}}\right)}^{2} \leq C\|\boldsymbol{v}\|_{L^{\infty}\left(0, T ; \boldsymbol{H}^{\theta}\right)}\|\boldsymbol{v}\|_{L^{2}\left(0, T ; \boldsymbol{H}^{1+\theta}\right)}$. 
(ii) Let $0<\theta \leq 1$ if $\boldsymbol{v} \in L^{\infty}\left(0, T ; \boldsymbol{H}^{\theta}\right)$, then $\boldsymbol{v} \otimes \boldsymbol{v} \in L^{\infty}\left(0, T ; H^{2 \theta-\frac{3}{2}}\left(\mathbb{T}_{3}\right)^{3 \times 3}\right)$ and there exists a constant $C>0$ such that

$$
\|\boldsymbol{v} \otimes \boldsymbol{v}\|_{L^{\infty}\left(0, T ; H^{2 \theta-\frac{3}{2}\left(\mathbb{T}_{3}\right)^{3 \times 3}}\right)} \leq C\|\boldsymbol{v}\|_{L^{\infty}\left(0, T ; \boldsymbol{H}^{\theta}\right)}^{2} .
$$

(iii) Let $0<\theta \leq 1$. If $\boldsymbol{v} \in L^{\infty}\left(0, T ; \boldsymbol{H}^{\theta}\right)$ and $\boldsymbol{w} \in L^{2}\left(0, T ; \boldsymbol{H}^{1}\right)$, then $\boldsymbol{v} \otimes \boldsymbol{w} \in$ $L^{2}\left(0, T ; H^{\theta-\frac{1}{2}}\left(\mathbb{T}_{3}\right)^{3 \times 3}\right)$ and there exists a constant $C>0$ such that

$$
\left.\|\boldsymbol{v} \otimes \boldsymbol{v}\|_{L^{2}\left(0, T ; H^{\theta-\frac{1}{2}\left(\mathbb{T}_{3}\right)}\right.}{ }^{3 \times 3}\right) \leq C\|\boldsymbol{v}\|_{L^{\infty}\left(0, T ; \boldsymbol{H}^{\theta}\right)}\|\boldsymbol{w}\|_{L^{2}\left(0, T ; \boldsymbol{H}^{1}\right)} .
$$

(iv) If $\boldsymbol{v} \in L^{\infty}\left(0, T ; \boldsymbol{L}^{2}\right) \cap L^{2}\left(0, T ; \boldsymbol{H}^{1}\right)$, then $\boldsymbol{v} \otimes \boldsymbol{v} \in L^{\frac{6}{5}}\left(0, T ; H^{\frac{1}{6}}\left(\mathbb{T}_{3}\right)^{3 \times 3}\right)$ and there exists a constant $C>0$ such that

$$
\begin{aligned}
\|\boldsymbol{v} \otimes \boldsymbol{v}\|_{L^{\frac{6}{5}}\left(0, T ; H^{\frac{1}{6}}\left(\mathbb{T}_{3}\right)^{3 \times 3}\right)} & \leq C\|\boldsymbol{v}\|_{L^{3}\left(0, T ; \boldsymbol{H}^{\frac{2}{3}}\right)}\|\boldsymbol{v}\|_{L^{2}\left(0, T ; \boldsymbol{H}^{1}\right)} \\
& \leq C\|\boldsymbol{v}\|_{L^{\infty}\left(0, T ; \boldsymbol{L}^{2}\right)}^{\frac{1}{3}}\|\boldsymbol{v}\|_{L^{2}\left(0, T ; \boldsymbol{H}^{1}\right)}^{\frac{5}{3}}
\end{aligned}
$$

Proof. Parts (ii) and (iii) are deduced from Lemma 2.2. Parts (i) and (iv) are obtained by using classical interpolation inequalities [1] combined with Lemma 2.2.

REMARK 2.1. When $\theta=1$ in Lemma 2.3-(i), we have $\boldsymbol{v} \in L^{\infty}\left(0, T ; \boldsymbol{H}^{1}\right) \cap$ $L^{2}\left(0, T ; \boldsymbol{H}^{2}\right) . \quad$ Since $\boldsymbol{v} \in L^{\infty}\left(0, T ; \boldsymbol{H}^{1}\right) \hookrightarrow L^{\infty}\left(0, T ; \boldsymbol{L}^{6}\right)$ and $L^{2}\left(0, T ; \boldsymbol{H}^{2}\right) \hookrightarrow$ $L^{2}\left(0, T ; \boldsymbol{L}^{\infty}\right)$, we deduce that $\boldsymbol{v} \otimes \boldsymbol{v} \in L^{2}\left(0, T ; L^{6}\left(\mathbb{T}_{3}\right)^{3 \times 3}\right)$ and there exists a constant $C>0$ such that

$$
\|\boldsymbol{v} \otimes \boldsymbol{v}\|_{L^{2}\left(0, T ; L^{6}\left(\mathbb{T}_{3}\right)^{3 \times 3}\right)} \leq C\|\boldsymbol{v}\|_{L^{\infty}\left(0, T ; \boldsymbol{H}^{1}\right)}\|\boldsymbol{v}\|_{L^{2}\left(0, T ; \boldsymbol{H}^{2}\right)} .
$$

3. Regular weak solution in the double viscous case. In this section we prove Theorem 1.1 by using the Galerkin method. For simplicity we will consider only the case when $0<N<\infty$ and the case $N=0$ is deduced by replacing $D_{0, \theta}$ by $I$. We start by constructing approximated solutions $\left(\boldsymbol{w}^{n}, \boldsymbol{B}^{n}, q^{n}\right)$ via Galerkin method. Then we seek for a priori estimates that are uniform with respect to $n$. Next, we passe to the limit in the equations after having used compactness properties. Finally we show that the solution we constructed is unique thanks to Gronwall's lemma [18].

Step 1 (Galerkin approximation). Consider a sequence $\left\{\varphi^{r}\right\}_{r=1}^{\infty}$ consisting of $L^{2}$-orthonormal and $H^{1}$-orthogonal eigenvectors of the Stokes problem subjected to the space periodic conditions. We note that this sequence forms a hilbertian basis of $L^{2}$.

We set

$$
\begin{aligned}
& \boldsymbol{w}^{n}(t, \boldsymbol{x})=\sum_{r=1}^{n} \boldsymbol{c}_{r}^{n}(t) \boldsymbol{\varphi}^{r}(\boldsymbol{x}), \quad \boldsymbol{B}^{n}(t, \boldsymbol{x})=\sum_{r=1}^{n} \boldsymbol{d}_{r}^{n}(t) \boldsymbol{\varphi}^{r}(\boldsymbol{x}), \\
& \text { and } q^{n}(t, \boldsymbol{x})=\sum_{|\boldsymbol{k}|=1}^{n} q_{\boldsymbol{k}}^{n}(t) e^{i \boldsymbol{k} \cdot \boldsymbol{x}} .
\end{aligned}
$$


We look for $\left(\boldsymbol{w}^{n}(t, \boldsymbol{x}), \boldsymbol{B}^{n}(t, \boldsymbol{x}), q^{n}(t, \boldsymbol{x})\right)$ that are determined through the system of equations

$$
\begin{array}{r}
\left(\partial \boldsymbol{w}^{n}, \varphi^{r}\right)-\left(\overline{D_{N, \theta}\left(\boldsymbol{w}^{n}\right) \otimes D_{N, \theta}\left(\boldsymbol{w}^{n}\right)}, \nabla \boldsymbol{\varphi}^{r}\right)+\nu\left(\nabla \boldsymbol{w}^{n}, \nabla \boldsymbol{\varphi}^{r}\right) \\
+\left(\overline{\boldsymbol{B}^{n} \otimes \boldsymbol{B}^{n}}, \nabla \boldsymbol{\varphi}^{r}\right)=0, \quad r=1,2, \ldots, n, \\
\left(\partial_{t} \boldsymbol{B}^{n}, \boldsymbol{\varphi}^{r}\right)+\left(D_{N, \theta}\left(\boldsymbol{w}^{n}\right) \otimes \boldsymbol{B}^{n}, \nabla \boldsymbol{\varphi}^{r}\right)+\mu\left(\nabla \boldsymbol{B}^{n}, \nabla \boldsymbol{\varphi}^{r}\right) \\
-\left(\boldsymbol{B}^{n} \otimes D_{N, \theta}\left(\boldsymbol{w}^{n}\right), \nabla \boldsymbol{\varphi}^{r}\right)=0, \quad r=1,2, \ldots, n,
\end{array}
$$

and

$$
\Delta q^{n}=-\nabla \cdot \nabla \cdot\left(\Pi^{n}\left(\overline{D_{N, \theta}\left(\boldsymbol{w}^{n}\right) \otimes D_{N, \theta}\left(\boldsymbol{w}^{n}\right)}-\overline{\boldsymbol{B}^{n} \otimes \boldsymbol{B}^{n}}\right)\right) .
$$

Where the projector $\Pi^{n}$ assign to any Fourier series $\sum_{\boldsymbol{k} \in \mathbb{Z}^{3} \backslash\{0\}} \boldsymbol{g}_{\boldsymbol{k}} e^{i \boldsymbol{k} \cdot \boldsymbol{x}}$ the following series $\sum_{\boldsymbol{k} \in \mathbb{Z}^{3} \backslash\{0\},|\boldsymbol{k}| \leq n} \boldsymbol{g}_{\boldsymbol{k}} e^{i \boldsymbol{k} \cdot \boldsymbol{x}}$.

Moreover we require that $\boldsymbol{w}^{n}$ and $\boldsymbol{B}^{n}$ satisfy the following initial conditions

$$
\boldsymbol{w}^{n}(0, .)=\boldsymbol{w}_{0}^{n}=\sum_{r=1}^{n} \boldsymbol{c}_{0}^{n} \boldsymbol{\varphi}^{r}(\boldsymbol{x}), \quad \boldsymbol{B}^{n}(0, .)=\boldsymbol{B}_{0}^{n}=\sum_{r=1}^{n} \boldsymbol{d}_{0}^{n} \boldsymbol{\varphi}^{r}(\boldsymbol{x})
$$

and

$$
\begin{array}{ccc}
\boldsymbol{w}_{0}^{n} \rightarrow \boldsymbol{w}_{0} & \text { strongly in } \boldsymbol{H}_{\sigma}^{\theta} & \text { when } n \rightarrow \infty, \\
\boldsymbol{B}_{0}^{n} \rightarrow \boldsymbol{B}_{0} & \text { strongly in } \boldsymbol{L}_{\sigma}^{2} & \text { when } n \rightarrow \infty
\end{array}
$$

The classical Caratheodory theory [19] then implies the short-time existence of solutions to (3.2)-(3.4). Next we derive estimates on $\boldsymbol{c}^{n}$ and $\boldsymbol{d}^{n}$ that are uniform w.r.t. $n$. These estimates then imply that the solution of (3.2)-(3.4) constructed on a short time interval $\left[0, T^{n}\right.$ [ exists for all $t \in[0, T]$.

Step 2 (A priori estimates). Multiplying the $r$ th equation in (3.2) with $\alpha^{2 \theta}|\boldsymbol{k}|^{2 \theta} \widehat{D_{N, \theta}} \boldsymbol{c}_{r}^{n}(t)+\widehat{D_{N, \theta}} \boldsymbol{c}_{r}^{n}(t)$, and the $r$ th equation in (3.3) with $\boldsymbol{d}_{r}^{n}(t)$, summing over $r=1,2, \ldots, n$, integrating over time from 0 to $t$ and using standard identities $[4,5]$ lead to the following equality

$$
\begin{aligned}
& \frac{1}{2}\left(\left\|\mathbb{A}_{\theta}^{\frac{1}{2}} D_{N, \theta}^{\frac{1}{2}}\left(\boldsymbol{w}^{n}\right)\right\|_{2}^{2}+\left\|\boldsymbol{B}^{n}\right\|_{2}^{2}\right)+\int_{0}^{t}\left(\nu\left\|\mathbb{A}_{\theta}^{\frac{1}{2}} D_{N, \theta}^{\frac{1}{2}} \boldsymbol{w}^{n}\right\|_{1,2}^{2}+\mu\left\|\boldsymbol{B}^{n}\right\|_{1,2}^{2}\right) d s \\
& =\frac{1}{2}\left(\left\|\mathbb{A}_{\theta}^{\frac{1}{2}} D_{N, \theta}^{\frac{1}{2}} \overline{\boldsymbol{v}}_{0}^{n}\right\|_{2}^{2}+\left\|\boldsymbol{B}_{0}^{n}\right\|_{2}^{2}\right) .
\end{aligned}
$$

Using (2.12) we conclude from (3.7) that

$$
\begin{aligned}
& \sup _{t \in\left[0, T^{n}[\right.}\left(\left\|\mathbb{A}_{\theta}^{\frac{1}{2}} D_{N, \theta}^{\frac{1}{2}}\left(\boldsymbol{w}^{n}\right)\right\|_{2}^{2}+\left\|\boldsymbol{B}^{n}\right\|_{2}^{2}\right) \\
& \quad+2 \int_{0}^{t}\left(\nu\left\|\mathbb{A}_{\theta}^{\frac{1}{2}} D_{N, \theta}^{\frac{1}{2}}\left(\boldsymbol{w}^{n}\right)\right\|_{1,2}^{2}+\mu\left\|\boldsymbol{B}^{n}\right\|_{1,2}^{2}\right) d s \leq\left\|\boldsymbol{v}_{0}^{n}\right\|_{2}^{2}+\left\|\boldsymbol{B}_{0}^{n}\right\|_{2}^{2} .
\end{aligned}
$$


The above inequality immediately implies that the existence time is independent of $n$ and it is possible to take $T=T^{n}$.

We deduce from (3.8) and (2.13) that

$$
\begin{array}{r}
\boldsymbol{w}^{n} \in L^{\infty}\left(0, T ; \boldsymbol{H}_{\sigma}^{\theta}\right) \cap L^{2}\left(0, T ; \boldsymbol{H}_{\sigma}^{1+\theta}\right), \\
\boldsymbol{B}^{n} \in L^{\infty}\left(0, T ; \boldsymbol{L}_{\sigma}^{2}\right) \cap L^{2}\left(0, T ; \boldsymbol{H}_{\sigma}^{1}\right) .
\end{array}
$$

Thus it follow from (2.10) that

$$
D_{N, \theta}\left(\boldsymbol{w}^{n}\right) \in L^{\infty}\left(0, T ; \boldsymbol{H}_{\sigma}^{\theta}\right) \cap L^{2}\left(0, T ; \boldsymbol{H}_{\sigma}^{1+\theta}\right) .
$$

From (3.10), (3.9) and by using Hölder inequality combined with Sobolev injection we get

$$
\begin{array}{r}
\boldsymbol{B}^{n} \otimes \boldsymbol{B}^{n} \in L^{2}\left(0, T ; H^{-\frac{1}{2}}\left(\mathbb{T}_{3}\right)^{3 \times 3}\right), \\
D_{N, \theta}\left(\boldsymbol{w}^{n}\right) \otimes \boldsymbol{B}^{n} \in L^{2}\left(0, T ; L^{2}\left(\mathbb{T}_{3}\right)^{3 \times 3}\right), \text { for any } \theta \geq \frac{1}{2}, \\
\boldsymbol{B}^{n} \otimes D_{N, \theta}\left(\boldsymbol{w}^{n}\right) \in L^{2}\left(0, T ; L^{2}\left(\mathbb{T}_{3}\right)^{3 \times 3}\right) \text { for any } \theta \geq \frac{1}{2} .
\end{array}
$$

Furthermore, from (3.10) and Lemma 2.3-(i) we also have,

$$
D_{N, \theta}\left(\boldsymbol{w}^{n}\right) \otimes D_{N, \theta}\left(\boldsymbol{w}^{n}\right) \in L^{2}\left(0, T ; H^{2 \theta-\frac{1}{2}}\left(\mathbb{T}_{3}\right)^{3 \times 3}\right) .
$$

From (3.12), (3.11) and (2.2) it follows that

$$
\begin{aligned}
\overline{\boldsymbol{B}^{n} \otimes \boldsymbol{B}^{n}} & \in L^{2}\left(0, T ; H^{2 \theta-\frac{1}{2}}\left(\mathbb{T}_{3}\right)^{3 \times 3}\right), \\
\overline{D_{N, \theta}\left(\boldsymbol{w}^{n}\right) \otimes D_{N, \theta}\left(\boldsymbol{w}^{n}\right)} & \in L^{2}\left(0, T ; H^{4 \theta-\frac{1}{2}}\left(\mathbb{T}_{3}\right)^{3 \times 3}\right) .
\end{aligned}
$$

Consequently from the elliptic theory (3.4) implies that

$$
\int_{0}^{T}\left\|q^{n}\right\|_{2 \theta-\frac{1}{2}, 2}^{2} d t<K
$$

From (3.2), (3.9), (3.13) and (3.14) we obtain that

$$
\int_{0}^{T}\left\|\partial_{t} \boldsymbol{w}^{n}\right\|_{2 \theta-\frac{3}{2}, 2}^{2} d t<K
$$

Finally, from (3.3), (3.9) and (3.11) we also obtain that

$$
\int_{0}^{T}\left\|\partial_{t} \boldsymbol{B}^{n}\right\|_{-1,2}^{2} d t<K
$$

Step 3 (Limit $n \rightarrow \infty$ ). It follows from the estimates (3.9)-(3.16) and the AubinLions compactness lemma (see [14] for example) that there are a not relabeled subsequence of $\left(\boldsymbol{w}^{n}, \boldsymbol{B}^{n}, q^{n}\right)$ and a triplet $(\boldsymbol{w}, \boldsymbol{B}, q)$ such that

$$
\begin{aligned}
\boldsymbol{w}^{n} \rightarrow \boldsymbol{w} \text { and } D_{N, \theta}\left(\boldsymbol{w}^{n}\right) & \rightarrow D_{N, \theta}(\boldsymbol{w}) & & \text { weakly in } L^{2}\left(0, T ; \boldsymbol{H}_{\sigma}^{1+\theta}\right), \\
\boldsymbol{B}^{n} & \rightarrow \boldsymbol{B} & & \text { weakly in } L^{2}\left(0, T ; \boldsymbol{H}_{\sigma}^{1}\right), \\
\partial_{t} \boldsymbol{w}^{n} & \rightarrow \partial_{t} \boldsymbol{w} & & \text { weakly in } L^{2}\left(0, T ; \boldsymbol{H}^{2 \theta-\frac{3}{2}}\right), \\
\partial_{t} \boldsymbol{B}^{n} & \rightarrow \partial_{t} \boldsymbol{B} & & \text { weakly in } L^{2}\left(0, T ; \boldsymbol{H}^{-1}\right), \\
q^{n} & \rightarrow q & & \text { weakly in } L^{2}\left(0, T ; H^{2 \theta-\frac{1}{2}}\left(\mathbb{T}_{3}\right)\right), \\
\boldsymbol{w}^{n} \rightarrow \boldsymbol{w} \text { and } D_{N, \theta}\left(\boldsymbol{w}^{n}\right) & \rightarrow D_{N, \theta}(\boldsymbol{w}) & & \text { strongly in } L^{2}\left(0, T ; \boldsymbol{H}_{\sigma}^{\theta}\right), \\
\boldsymbol{B}^{n} & \rightarrow \boldsymbol{B} & & \text { strongly in } L^{2}\left(0, T ; \boldsymbol{L}_{\sigma}^{2}\right) .
\end{aligned}
$$


From (3.17) and (3.22) it follows that

$\left.\overline{D_{N, \theta}\left(\boldsymbol{w}^{n}\right) \otimes D_{N, \theta}\left(\boldsymbol{w}^{n}\right.}\right) \rightarrow \overline{D_{N, \theta}(\boldsymbol{w}) \otimes D_{N, \theta}(\boldsymbol{w})}$ strongly in $L^{1}\left(0, T ; L^{1}\left(\mathbb{T}_{3}\right)^{3 \times 3}\right)$.

From (3.18) and (3.22) it follows that

$$
\begin{array}{ll}
\boldsymbol{B}^{n} \otimes D_{N, \theta}\left(\boldsymbol{w}^{n}\right) \rightarrow \boldsymbol{B} \otimes \boldsymbol{w} & \text { strongly in } L^{1}\left(0, T ; L^{1}\left(\mathbb{T}_{3}\right)^{3 \times 3}\right), \\
D_{N, \theta}\left(\boldsymbol{w}^{n}\right) \otimes \boldsymbol{B}^{n} \rightarrow \boldsymbol{w} \otimes \boldsymbol{B} & \text { strongly in } L^{1}\left(0, T ; L^{1}\left(\mathbb{T}_{3}\right)^{3 \times 3}\right) .
\end{array}
$$

From (3.18) and (3.23) it follows that

$$
\overline{\boldsymbol{B}^{n} \otimes \boldsymbol{B}^{n}} \rightarrow \overline{\boldsymbol{B} \otimes \boldsymbol{B}} \quad \text { strongly in } L^{1}\left(0, T ; L^{1}\left(\mathbb{T}_{3}\right)^{3 \times 3}\right) .
$$

Finally, since the sequence $\left\{\overline{D_{N, \theta}\left(\boldsymbol{w}^{n}\right) \otimes D_{N, \theta}\left(\boldsymbol{w}^{n}\right)}\right\}_{n \in \mathbb{N}}$ is bounded in $L^{2}\left(0, T ; H^{4 \theta-\frac{3}{2}}\left(\mathbb{T}_{3}\right)^{3 \times 3}\right)$, it converges weakly, up to a subsequence, to some $\chi$ in $L^{2}\left(0, T ; H^{4 \theta-\frac{3}{2}}\left(\mathbb{T}_{3}\right)^{3 \times 3}\right)$. The result above (3.24) and uniqueness of the limit, allows us to claim that $\chi=\overline{D_{N, \theta}(\boldsymbol{w}) \otimes D_{N, \theta}(\boldsymbol{w})}$. Consequently

$\left.\overline{D_{N, \theta}\left(\boldsymbol{w}^{n}\right) \otimes D_{N, \theta}\left(\boldsymbol{w}^{n}\right.}\right) \rightarrow \overline{D_{N, \theta}(\boldsymbol{w}) \otimes D_{N, \theta}(\boldsymbol{w})} \quad$ weakly in $L^{2}\left(0, T ; H^{4 \theta-\frac{3}{2}}\left(\mathbb{T}_{3}\right)^{3 \times 3}\right)$.

Similarly,

$$
\begin{aligned}
& D_{N, \theta}\left(\boldsymbol{w}^{n}\right) \otimes \boldsymbol{B}^{n} \rightarrow D_{N, \theta}(\boldsymbol{w}) \otimes \boldsymbol{B} \quad \text { weakly in } L^{2}\left(0, T ; L^{2}\left(\mathbb{T}_{3}\right)^{3 \times 3}\right) \text {, } \\
& \boldsymbol{B}^{n} \otimes D_{N, \theta}\left(\boldsymbol{w}^{n}\right) \rightarrow \boldsymbol{B} \otimes D_{N, \theta} \boldsymbol{w} \quad \text { weakly in } L^{2}\left(0, T ; L^{2}\left(\mathbb{T}_{3}\right)^{3 \times 3}\right) \text {, } \\
& \overline{\boldsymbol{B}^{n} \otimes \boldsymbol{B}^{n}}-\overline{\boldsymbol{B} \otimes \boldsymbol{B}} \quad \text { weakly in } L^{2}\left(0, T ; H^{2 \theta-\frac{1}{2}}\left(\mathbb{T}_{3}\right)^{3 \times 3}\right) \text {. }
\end{aligned}
$$

The above established convergences are clearly sufficient for taking the limit in (3.2)-(3.3) and for concluding that $\boldsymbol{w}$ and $\boldsymbol{B}$ satisfy (1.7)-(1.8).

Note that since $\overline{\boldsymbol{B} \otimes \boldsymbol{B}} \in L^{2}\left(0, T ; H^{2 \theta-\frac{1}{2}}\left(\mathbb{T}_{3}\right)^{3 \times 3}\right)$, then $\partial_{t} \boldsymbol{w} \in$ $L^{2}\left(0, T ; H^{2 \theta-\frac{1}{2}}\left(\mathbb{T}_{3}\right)^{3 \times 3}\right)$. Therefore, we have

$$
\partial_{t} \boldsymbol{w} \in L^{2}\left(0, T ; H^{\theta-1}\left(\mathbb{T}_{3}\right)^{3 \times 3}\right) \text { for all } \theta \geq \frac{1}{2} .
$$

When $\theta=1$, we also conclude that $\partial_{t} \boldsymbol{w} \in L^{2}\left(0, T ; H^{\frac{1}{2}}\left(\mathbb{T}_{3}\right)^{3 \times 3}\right)$ which is better than the estimation found in [4].

Moreover, from (3.17) and (3.29) one can deduce by a classical argument (see [1] for instance) that

$$
\boldsymbol{w} \in \mathcal{C}\left(0, T ; \boldsymbol{H}_{\sigma}^{\theta}\right){ }^{1}
$$

Similarly, we deduce from (3.18) and (3.20) that

$$
\boldsymbol{B} \in \mathcal{C}\left(0, T ; \boldsymbol{L}_{\sigma}^{2}\right) .
$$

Furthermore, from the strong continuity of $\boldsymbol{w}$ and $\boldsymbol{B}$ with respect to the time with value in $\boldsymbol{H}^{\theta}$ and $\boldsymbol{L}_{\sigma}^{2}$ respectively, we deduce that $\boldsymbol{w}(0)=\boldsymbol{w}_{0}$ and $\boldsymbol{B}(0)=\boldsymbol{B}_{0}$.

\footnotetext{
${ }^{1}$ Note that from (3.17) and (3.19), we also deduce that $\boldsymbol{w} \in \mathcal{C}\left(0, T ; \boldsymbol{H}_{\sigma}^{\frac{3 \theta}{2}-\frac{1}{4}}\right)$. However, $\frac{3 \theta}{2}-\frac{1}{4}$ is equal to $\theta$ when $\theta=\frac{1}{2}$.
} 
Let us mention also that for $\theta \geq \frac{1}{2}, D_{N, \theta}(\boldsymbol{w})+\alpha^{2 \theta}(-\Delta)^{\theta} D_{N, \theta}(\boldsymbol{w}) \in L^{2}\left(0, T ; \boldsymbol{H}_{\sigma}^{1-\theta}\right) \hookrightarrow$ $L^{2}\left(0, T ; \boldsymbol{H}_{\sigma}^{\frac{3}{2}-2 \theta}\right)$ and $\boldsymbol{B} \in L^{2}\left(0, T ; \boldsymbol{H}_{\sigma}^{1}\right)$ hence $\mathbb{A}_{\theta} D_{N, \theta}(\boldsymbol{w})$ is a possible test function in the weak formulation (1.7) and $\boldsymbol{B}$ is a possible test function in the weak formulation (1.8). Thus $\mathbb{A}_{\theta}^{\frac{1}{2}} D_{N, \theta}^{\frac{1}{2}}(\boldsymbol{w})$ and $\boldsymbol{B}$ verify for all $t \in[0, T]$ the following equality

$$
\begin{aligned}
& \frac{1}{2}\left(\left\|\mathbb{A}_{\theta}^{\frac{1}{2}} D_{N, \theta}^{\frac{1}{2}}(\boldsymbol{w})\right\|_{2}^{2}+\|\boldsymbol{B}\|_{2}^{2}\right)+\int_{0}^{t}\left(\nu\left\|\mathbb{A}_{\theta}^{\frac{1}{2}} D_{N, \theta}^{\frac{1}{2}}(\boldsymbol{w})\right\|_{1,2}^{2}+\mu\|\boldsymbol{B}\|_{1,2}^{2}\right) d s \\
& =\frac{1}{2}\left(\left\|\mathbb{A}_{\theta}^{\frac{1}{2}} D_{N, \theta}^{\frac{1}{2}}\left(\overline{\boldsymbol{v}}_{0}\right)\right\|_{2}^{2}+\left\|\boldsymbol{B}_{0}\right\|_{2}^{2}\right) .
\end{aligned}
$$

Step 4 (Uniqueness). Next, we will show the continuous dependence of the solutions on the initial data and in particular the uniqueness.

The proof of this result is an adaptation of the uniqueness result which is given for the Rotational Approximate Deconvolution Model (RADM) in [3]. ${ }^{2}$

For simplicity, we restrict ourselves to the critical case $\theta=\frac{1}{2}$, and we drop some indices of $\theta$. Thus we will write " $D_{N}$ " instead of " $D_{N, \theta}$ " and "A" instead of " $\mathbb{A}_{\theta}$ " expecting that no confusion will occur.

Let $\left(\boldsymbol{w}_{1}, \boldsymbol{B}_{1}, q_{1}\right)$ and $\left(\boldsymbol{w}_{2}, \boldsymbol{B}_{2}, q_{2}\right)$ be any two solutions of (1.1) on the interval [0,T], with initial values $\left(\boldsymbol{w}_{1}(0), \boldsymbol{B}_{1}(0)\right)$ and $\left(\boldsymbol{w}_{2}(0), \boldsymbol{B}_{2}(0)\right)$. Let us denote by $\delta \boldsymbol{w}=\boldsymbol{w}_{2}-$ $\boldsymbol{w}_{1}$, by $\delta \boldsymbol{B}=\boldsymbol{B}_{2}-\boldsymbol{B}_{1}$ and by $\delta q=q_{2}-q_{1}$.

Then one has

$$
\begin{array}{r}
\left.\partial_{t} \delta \boldsymbol{w}-\nu \Delta \delta \boldsymbol{w}+\nabla \cdot\left(\overline{D_{N}\left(\boldsymbol{w}_{2}\right) \otimes D_{N}\left(\boldsymbol{w}_{2}\right)}\right)-\nabla \cdot\left(\overline{D_{N}\left(\boldsymbol{w}_{1}\right) \otimes D_{N}\left(\boldsymbol{w}_{1}\right.}\right)\right) \\
-\nabla \cdot\left(\overline{\boldsymbol{B}_{2} \otimes \boldsymbol{B}_{2}}\right)+\nabla \cdot\left(\overline{\boldsymbol{B}_{1} \otimes \boldsymbol{B}_{1}}\right)+\nabla \delta q=0 \\
\partial_{t} \delta \boldsymbol{B}-\mu \Delta \delta \boldsymbol{B}+\nabla \cdot\left(D_{N}\left(\boldsymbol{w}_{2}\right) \otimes \boldsymbol{B}_{2}\right)-\nabla \cdot\left(D_{N}\left(\boldsymbol{w}_{1}\right) \otimes \boldsymbol{B}_{1}\right) \\
-\nabla \cdot\left(\boldsymbol{B}_{2} \otimes D_{N}\left(\boldsymbol{w}_{2}\right)\right)+\nabla \cdot\left(\boldsymbol{B}_{1} \otimes D_{N}\left(\boldsymbol{w}_{1}\right)\right)=0
\end{array}
$$

and $\delta \boldsymbol{w}=0, \delta \boldsymbol{B}=0$ at initial time. One can take $\alpha(-\Delta)^{\frac{1}{2}} D_{N}(\delta \boldsymbol{w})+D_{N}(\delta \boldsymbol{w})$ as test in the first equation of (3.30) and $\delta \boldsymbol{B}$ as test in the second equations of (3.30). Since $D_{N}\left(\boldsymbol{w}_{1}\right)$ is divergence-free we have

$$
\begin{aligned}
& \int_{0}^{T} \int_{\mathbb{T}_{3}} D_{N}\left(\boldsymbol{w}_{1}\right) \otimes D_{N}(\delta \boldsymbol{w}): \nabla D_{N}(\delta \boldsymbol{w}) \\
& =-\int_{0}^{T} \int_{\mathbb{T}_{3}}\left(D_{N}\left(\boldsymbol{w}_{1}\right) \cdot \nabla\right) D_{N}(\delta \boldsymbol{w}) \cdot D_{N}(\delta \boldsymbol{w})=0 .
\end{aligned}
$$

Thus we obtain by using the fact that the averaging operator commutes with differ-

\footnotetext{
${ }^{2}$ This proof is slightly different from the corresponding uniqueness result for the MHD Deconvolution Model in [4] given that we use the inequality which satisfied by $\boldsymbol{w}$ instead of $D_{N, \theta}(\boldsymbol{w})$. For this reason, we will get the term $(N+1)^{6}$ instead of $(N+1)^{4}$ in [4], (see inequality (3.44)). However, in both cases, it is not possible to pass to the limit $N \rightarrow \infty$ in the inequality (3.44).
} 
entiation under periodic boundary conditions

$$
\begin{aligned}
& \int_{0}^{T} \int_{\mathbb{T}_{3}}\left(\nabla \cdot\left(\overline{D_{N}\left(\boldsymbol{w}_{2}\right) \otimes D_{N}\left(\boldsymbol{w}_{2}\right)}\right)-\nabla \cdot\left(\overline{D_{N}\left(\boldsymbol{w}_{1}\right) \otimes D_{N}\left(\boldsymbol{w}_{1}\right)}\right)\right) \\
& \quad \cdot\left(\alpha(-\Delta)^{\frac{1}{2}} D_{N}(\delta \boldsymbol{w})+D_{N}(\delta \boldsymbol{w})\right) \\
& =\int_{0}^{T} \int_{\mathbb{T}_{3}}\left(\nabla \cdot\left(D_{N}\left(\boldsymbol{w}_{2}\right) \otimes D_{N}\left(\boldsymbol{w}_{2}\right)\right)-\nabla \cdot\left(D_{N}\left(\boldsymbol{w}_{1}\right) \otimes D_{N}\left(\boldsymbol{w}_{1}\right)\right)\right) \cdot D_{N}(\delta \boldsymbol{w}) \\
& =-\int_{0}^{T} \int_{\mathbb{T}_{3}} D_{N}(\delta \boldsymbol{w}) \otimes D_{N}\left(\boldsymbol{w}_{2}\right): \nabla D_{N}(\delta \boldsymbol{w}) .
\end{aligned}
$$

Similarly, because $D_{N}\left(\boldsymbol{w}_{1}\right)$ is divergence-free we have

$$
\int_{0}^{T} \int_{\mathbb{T}_{3}} D_{N}\left(\boldsymbol{w}_{1}\right) \otimes \delta \boldsymbol{B}: \nabla \delta \boldsymbol{B}=-\int_{0}^{T} \int_{\mathbb{T}_{3}}\left(D_{N}\left(\boldsymbol{w}_{1}\right) \cdot \nabla\right) \delta \boldsymbol{B} \cdot \delta \boldsymbol{B}=0,
$$

and thus we have the following identity

$$
\begin{aligned}
& \int_{0}^{T} \int_{\mathbb{T}_{3}}\left(\nabla \cdot\left(D_{N}\left(\boldsymbol{w}_{2}\right) \otimes \boldsymbol{B}_{2}\right)-\nabla \cdot\left(D_{N}\left(\boldsymbol{w}_{1}\right) \otimes \boldsymbol{B}_{1}\right)\right) \cdot \delta \boldsymbol{B} \\
& =\int_{0}^{T} \int_{\mathbb{T}_{3}}\left(\nabla \cdot\left(D_{N}\left(\boldsymbol{w}_{2}\right) \otimes \boldsymbol{B}_{2}\right)-\nabla \cdot\left(D_{N}\left(\boldsymbol{w}_{1}\right) \otimes \boldsymbol{B}_{1}\right)\right) \cdot \delta \boldsymbol{B} \\
& =-\int_{0}^{T} \int_{\mathbb{T}_{3}} D_{N}(\delta \boldsymbol{w}) \otimes \boldsymbol{B}_{2}: \nabla \delta \boldsymbol{B} .
\end{aligned}
$$

Concerning the remaining terms we get by integrations by parts and by using the fact that the averaging operator commutes with differentiation under periodic boundary conditions

$$
\begin{aligned}
& \int_{0}^{T} \int_{\mathbb{T}_{3}}\left(-\nabla \cdot\left(\overline{\boldsymbol{B}_{2} \otimes \boldsymbol{B}_{2}}\right)+\nabla \cdot\left(\overline{\boldsymbol{B}_{1} \otimes \boldsymbol{B}_{1}}\right)\right) \cdot\left(\alpha(-\Delta)^{\frac{1}{2}} D_{N}(\delta \boldsymbol{w})+D_{N}(\delta \boldsymbol{w})\right) \\
& =\int_{0}^{T} \int_{\mathbb{T}_{3}}\left(-\nabla \cdot\left(\boldsymbol{B}_{2} \otimes \boldsymbol{B}_{2}\right)+\nabla \cdot\left(\boldsymbol{B}_{1} \otimes \boldsymbol{B}_{1}\right)\right) \cdot D_{N}(\delta \boldsymbol{w}) \\
& =\int_{0}^{T} \int_{\mathbb{T}_{3}} \boldsymbol{B}_{1} \otimes \delta \boldsymbol{B}: \nabla D_{N}(\delta \boldsymbol{w})+\delta \boldsymbol{B} \otimes \boldsymbol{B}_{2}: \nabla D_{N}(\delta \boldsymbol{w}) .
\end{aligned}
$$

Similarly,

$$
\begin{aligned}
& \int_{0}^{T} \int_{\mathbb{T}_{3}}\left(-\nabla \cdot\left(\boldsymbol{B}_{2} \otimes D_{N}\left(\boldsymbol{w}_{2}\right)\right)+\nabla \cdot\left(\boldsymbol{B}_{1} \otimes D_{N}\left(\boldsymbol{w}_{1}\right)\right)\right) \cdot \delta \boldsymbol{B} \\
& =\int_{0}^{T} \int_{\mathbb{T}_{3}}\left(\nabla \cdot\left(\boldsymbol{B}_{2} \otimes D_{N}\left(\boldsymbol{w}_{2}\right)\right)-\nabla \cdot\left(\boldsymbol{B}_{1} \otimes D_{N}\left(\boldsymbol{w}_{1}\right)\right)\right) \cdot \delta \boldsymbol{B} \\
& =\int_{0}^{T} \int_{\mathbb{T}_{3}}-\left(\boldsymbol{B}_{1} \cdot \nabla\right) D_{N}(\delta \boldsymbol{w}) \cdot \delta \boldsymbol{B}+\delta \boldsymbol{B} \otimes D_{N}\left(\boldsymbol{w}_{2}\right): \nabla \delta \boldsymbol{B} .
\end{aligned}
$$

Therefore by adding (3.32)-(3.36) and using the fact that the averaging operator 
commutes with differentiation under periodic boundary conditions we obtain

$$
\begin{aligned}
& \frac{1}{2} \frac{d}{d t}\left(\left\|\mathbb{A}^{\frac{1}{2}} D_{N}^{\frac{1}{2}}(\delta \boldsymbol{w})\right\|_{2}^{2}+\|\delta \boldsymbol{B}\|_{2}^{2}\right)+\nu\left\|\nabla \mathbb{A}^{\frac{1}{2}} D_{N}^{\frac{1}{2}}(\delta \boldsymbol{w})\right\|_{2}^{2}+\mu\|\delta \boldsymbol{B}\|_{1,2}^{2} \\
& =\int_{\mathbb{T}_{3}} D_{N}(\delta \boldsymbol{w}) \otimes D_{N}\left(\boldsymbol{w}_{2}\right): \nabla D_{N}(\delta \boldsymbol{w})+\int_{\mathbb{T}_{3}} D_{N}(\delta \boldsymbol{w}) \otimes \boldsymbol{B}_{2}: \nabla \delta \boldsymbol{B} \\
& \quad-\int_{\mathbb{T}_{3}} \delta \boldsymbol{B} \otimes \boldsymbol{B}_{2}: \nabla D_{N}(\delta \boldsymbol{w})-\int_{\mathbb{T}_{3}} \delta \boldsymbol{B} \otimes D_{N}\left(\boldsymbol{w}_{2}\right): \nabla \delta \boldsymbol{B} .
\end{aligned}
$$

Next, we estimate the four integrals in the right hand side of (3.37). The estimates are obtained by using Hölder inequality, Sobolev embedding theorem, Young inequality and Lemma 2.1 .

$$
\begin{aligned}
& \left|\int_{\mathbb{T}_{3}} D_{N}(\delta \boldsymbol{w}) \otimes D_{N}\left(\boldsymbol{w}_{2}\right): \nabla D_{N}(\delta \boldsymbol{w})\right| \\
& \leq \frac{C(N+1)^{2}}{\alpha \nu}\left\|D_{N}(\delta \boldsymbol{w}) \otimes D_{N}\left(\boldsymbol{w}_{2}\right)\right\|_{-\frac{1}{2}, 2}^{2}+\frac{\nu \alpha}{4}\|\nabla \delta \boldsymbol{w}\|_{\frac{1}{2}, 2}^{2} \\
& \leq \frac{C(N+1)^{6}}{\alpha \nu}\|\delta \boldsymbol{w}\|_{\frac{1}{2}, 2}^{2}\left\|\boldsymbol{w}_{2}\right\|_{\frac{3}{2}, 2}^{2}+\frac{\nu \alpha}{4}\|\nabla \delta \boldsymbol{w}\|_{\frac{1}{2}, 2}^{2} \\
& \left|\int_{\mathbb{T}_{3}} D_{N}(\delta \boldsymbol{w}) \otimes \boldsymbol{B}_{2}: \nabla \delta \boldsymbol{B}\right| \leq \frac{C}{\mu}\left\|D_{N}(\delta \boldsymbol{w}) \otimes \boldsymbol{B}_{2}\right\|_{2}^{2}+\frac{\mu}{4}\|\nabla \delta \boldsymbol{B}\|_{2}^{2} \\
& \leq \frac{C(N+1)^{2}}{\mu}\|\delta \boldsymbol{w}\|_{\frac{1}{2}, 2}^{2}\left\|\boldsymbol{B}_{2}\right\|_{1,2}^{2}+\frac{\mu}{4}\|\nabla \delta \boldsymbol{B}\|_{2}^{2} \\
& \left|\int_{\mathbb{T}_{3}} \delta \boldsymbol{B} \otimes \boldsymbol{B}_{2}: \nabla D_{N}(\delta \boldsymbol{w})\right| \leq \frac{C(N+1)^{2}}{\alpha \nu}\left\|\delta \boldsymbol{B} \otimes \boldsymbol{B}_{2}\right\|_{-\frac{1}{2}, 2}^{2}+\frac{\nu \alpha}{4}\|\nabla \delta \boldsymbol{w}\|_{\frac{1}{2}, 2}^{2} \\
& \leq \frac{C(N+1)^{2}}{\alpha \nu}\|\delta \boldsymbol{B}\|_{2}^{2}\left\|\boldsymbol{B}_{2}\right\|_{1,2}^{2}+\frac{\nu \alpha}{4}\|\nabla \delta \boldsymbol{w}\|_{\frac{1}{2}, 2}^{2}, \\
& \left|\int_{\mathbb{T}_{3}} \delta \boldsymbol{B} \otimes D_{N}\left(\boldsymbol{w}_{2}\right): \nabla \delta \boldsymbol{B}\right|=\left|\int_{\mathbb{T}_{3}}(\delta \boldsymbol{B} \cdot \nabla) D_{N}\left(\boldsymbol{w}_{2}\right) \cdot \delta \boldsymbol{B}\right| \\
& \leq \frac{C}{\mu}\|\delta \boldsymbol{B}\|_{L^{2}}^{2}\left\|\nabla D_{N}\left(\boldsymbol{w}_{2}\right)\right\|_{L^{3}}^{2}+\frac{\mu}{4}\|\delta \boldsymbol{B}\|_{L^{6}}^{2} \\
& \leq \frac{C(N+1)^{2}}{\mu}\|\delta \boldsymbol{B}\|_{2}^{2}\left\|\boldsymbol{w}_{2}\right\|_{\frac{3}{2}, 2}^{2}+\frac{\mu}{4}\|\delta \boldsymbol{B}\|_{1,2}^{2} .
\end{aligned}
$$

By using (2.13) we have

$$
\begin{aligned}
& \frac{1}{2} \frac{d}{d t}\left(\alpha\|\delta \boldsymbol{w}\|_{\frac{1}{2}, 2}^{2}+\|\delta \boldsymbol{B}\|_{2}^{2}\right)+\nu \alpha\|\nabla \delta \boldsymbol{w}\|_{\frac{1}{2}}^{2}+\mu\|\delta \boldsymbol{B}\|_{1,2}^{2} \\
& \leq \frac{1}{2} \frac{d}{d t}\left(\left\|\mathbb{A}^{\frac{1}{2}} D_{N}^{\frac{1}{2}}(\delta \boldsymbol{w})\right\|_{2}^{2}+\|\delta \boldsymbol{B}\|_{2}^{2}\right)+\nu\left\|\nabla \mathbb{A}^{\frac{1}{2}} D_{N}^{\frac{1}{2}}(\delta \boldsymbol{w})\right\|_{2}^{2}+\mu\|\delta \boldsymbol{B}\|_{1,2}^{2}
\end{aligned}
$$

From (3.37)-(3.42) we get

$$
\begin{aligned}
& \frac{d}{d t}\left(\alpha\|\delta \boldsymbol{w}\|_{\frac{1}{2}, 2}^{2}+\|\delta \boldsymbol{B}\|_{2}^{2}\right)+\nu \alpha\|\nabla \delta \boldsymbol{w}\|_{\frac{1}{2}}^{2}+\mu\|\delta \boldsymbol{B}\|_{1,2}^{2} \\
& \leq \frac{C(N+1)^{6}}{\alpha \nu}\|\delta \boldsymbol{w}\|_{\frac{1}{2}, 2}^{2}\left\|\boldsymbol{w}_{2}\right\|_{\frac{3}{2}, 2}^{2}+\frac{C(N+1)^{2}}{\mu}\|\delta \boldsymbol{w}\|_{\frac{1}{2}, 2}^{2}\left\|\boldsymbol{B}_{2}\right\|_{1,2}^{2} \\
& \quad+\frac{C(N+1)^{2}}{\alpha \nu}\|\delta \boldsymbol{B}\|_{2}^{2}\left\|\boldsymbol{B}_{2}\right\|_{1,2}^{2}+\frac{C(N+1)^{2}}{\mu}\|\delta \boldsymbol{B}\|_{2}^{2}\left\|\boldsymbol{w}_{2}\right\|_{\frac{3}{2}, 2}^{2}
\end{aligned}
$$


Hence,

$$
\begin{aligned}
& \frac{d}{d t}\left(\alpha\|\delta \boldsymbol{w}\|_{\frac{1}{2}, 2}^{2}+\|\delta \boldsymbol{B}\|_{2}^{2}\right)+\nu \alpha\|\nabla \delta \boldsymbol{w}\|_{\frac{1}{2}}^{2}+\mu\|\delta \boldsymbol{B}\|_{1,2}^{2} \\
& \leq \frac{C(N+1)^{6}}{\min (\alpha \nu, \mu)}\left(\alpha\|\delta \boldsymbol{w}\|_{\frac{1}{2}, 2}^{2}+\|\delta \boldsymbol{B}\|_{2}^{2}\right)\left(\left\|\boldsymbol{w}_{2}\right\|_{\frac{3}{2}, 2}^{2}+\left\|\boldsymbol{B}_{2}\right\|_{1,2}^{2}\right) .
\end{aligned}
$$

Since $\left\|\boldsymbol{w}_{2}\right\|_{\frac{3}{2}, 2}^{2}+\left\|\boldsymbol{B}_{2}\right\|_{1,2}^{2} \in L^{1}([0, T])$, we get by using Gronwall's inequality the continuous dependence of the solutions on the initial data. In particular, if $\delta \boldsymbol{w}_{0}=$ $\delta \boldsymbol{B}_{0}=0$ then $\delta \boldsymbol{w}=\delta \boldsymbol{B}=0$ and the solutions are unique for all $t \in[0, T]$. Since $T>0$ is arbitrary this solution may be uniquely extended for all time.

This finishes the proof of Theorem 1.1.

4. Regular weak solution in the inviscid case $(\nu=0, \mu>0)$. In this section we prove Theorem 1.2 by using the Galerkin method.

Step 1: (Existence of weak solutions).

Step 1-i: (Galerkin approximation). Consider the sequence $\left\{\varphi^{r}\right\}_{r=1}^{\infty}$ defined in the proof of Theorem 1.1. We look for $\left(\boldsymbol{w}^{n}(t, \boldsymbol{x}), \boldsymbol{B}^{n}(t, \boldsymbol{x}), q^{n}(t, \boldsymbol{x})\right)$, where

$$
\begin{aligned}
\boldsymbol{w}^{n}(t, \boldsymbol{x})= & \sum_{r=1}^{n} \boldsymbol{c}_{r}^{n}(t) \boldsymbol{\varphi}^{r}(\boldsymbol{x}), \quad \boldsymbol{B}^{n}(t, \boldsymbol{x})=\sum_{r=1}^{n} \boldsymbol{d}_{r}^{n}(t) \boldsymbol{\varphi}^{r}(\boldsymbol{x}), \\
& \text { and } q^{n}(t, \boldsymbol{x})=\sum_{|\boldsymbol{k}|=1}^{n} q_{\boldsymbol{k}}^{n}(t) e^{i \boldsymbol{k} \cdot \boldsymbol{x}}
\end{aligned}
$$

that are determined through the system of equations

$$
\begin{gathered}
\left(\partial_{t} \boldsymbol{w}^{n}, \boldsymbol{\varphi}^{r}\right)-\left(\overline{D_{N, \theta}\left(\boldsymbol{w}^{n}\right) \otimes D_{N, \theta}\left(\boldsymbol{w}^{n}\right)}, \nabla \boldsymbol{\varphi}^{r}\right) \\
+\left(\overline{\boldsymbol{B}^{n} \otimes \boldsymbol{B}^{n}}, \nabla \boldsymbol{\varphi}^{r}\right)=0, \quad r=1,2, \ldots, n, \\
\left(\partial_{t} \boldsymbol{B}^{n}, \boldsymbol{\varphi}^{r}\right)+\left(D_{N, \theta}\left(\boldsymbol{w}^{n}\right) \otimes \boldsymbol{B}^{n}, \nabla \boldsymbol{\varphi}^{r}\right)+\mu\left(\nabla \boldsymbol{B}^{n}, \nabla \boldsymbol{\varphi}^{r}\right) \\
-\left(\boldsymbol{B}^{n} \otimes D_{N, \theta}\left(\boldsymbol{w}^{n}\right), \nabla \boldsymbol{\varphi}^{r}\right)=0, \quad r=1,2, \ldots, n,
\end{gathered}
$$

and

$$
\Delta q^{n}=-\nabla \cdot \nabla \cdot\left(\Pi^{n}\left(\overline{D_{N, \theta}\left(\boldsymbol{w}^{n}\right) \otimes D_{N, \theta}\left(\boldsymbol{w}^{n}\right)}-\overline{\boldsymbol{B}^{n} \otimes \boldsymbol{B}^{n}}\right)\right) .
$$

Where the projector $\Pi^{n}$ assign to any Fourier series $\sum_{\boldsymbol{k} \in \mathbb{Z}^{3} \backslash\{0\}} \boldsymbol{g}_{\boldsymbol{k}} e^{i \boldsymbol{k} \cdot \boldsymbol{x}}$ the following series $\sum_{\boldsymbol{k} \in \mathbb{Z}^{3} \backslash\{0\},|\boldsymbol{k}| \leq n} \boldsymbol{g}_{\boldsymbol{k}} e^{i \boldsymbol{k} \cdot \boldsymbol{x}}$.

Moreover we require that $\boldsymbol{w}^{n}$ and $\boldsymbol{B}^{n}$ satisfy the following initial conditions

$$
\boldsymbol{w}^{n}(0, .)=\boldsymbol{w}_{0}^{n}=\sum_{r=1}^{n} \boldsymbol{c}_{0}^{n} \boldsymbol{\varphi}^{r}(\boldsymbol{x}), \quad \boldsymbol{B}^{n}(0, .)=\boldsymbol{B}_{0}^{n}=\sum_{r=1}^{n} \boldsymbol{d}_{0}^{n} \boldsymbol{\varphi}^{r}(\boldsymbol{x})
$$

and

$$
\begin{array}{ccc}
\boldsymbol{w}_{0}^{n} \rightarrow \boldsymbol{w}_{0} & \text { strongly in } \boldsymbol{H}_{\sigma}^{2 \theta} & \text { when } n \rightarrow \infty, \\
\boldsymbol{B}_{0}^{n} \rightarrow \boldsymbol{B}_{0} & \text { strongly in } \boldsymbol{L}_{\sigma}^{2} & \text { when } n \rightarrow \infty
\end{array}
$$


The classical Caratheodory theory [19] then implies the short-time existence of solutions to (4.2)-(4.4). Next we derive estimates on $\boldsymbol{c}^{n}$ and $\boldsymbol{d}^{n}$ that are uniform w.r.t. $n$. These estimates then imply that the solution of (4.2)-(4.4) constructed on a short time interval $\left[0, T^{n}[\right.$ exists for all $t \in[0, T]$.

Step 1-ii: (Uniform estimates). We are going to derive some new uniform estimates on $\left(\boldsymbol{w}^{n}, \boldsymbol{B}^{n}, q^{n}\right)$.

Multiplying the $r$ th equation in (4.2) with $\alpha^{2 \theta}|\boldsymbol{k}|^{2 \theta} \widehat{D_{N, \theta}} \boldsymbol{c}_{r}^{n}(t)+\widehat{D_{N, \theta}} \boldsymbol{c}_{r}^{n}(t)$, and the $r$ th equation in (4.3) with $\boldsymbol{d}_{r}^{n}(t)$ summing over $r=1,2, \ldots, n$, integrating over time from 0 to $t$ we obtain in a similar way than in the proof of Theorem 1.1,

$$
\sup _{t \in[0, T]}\left(\left\|\mathbb{A}_{\theta}^{\frac{1}{2}} D_{N, \theta}^{\frac{1}{2}}\left(\boldsymbol{w}^{n}\right)\right\|_{2}^{2}+\left\|\boldsymbol{B}^{n}\right\|_{2}^{2}\right)+2 \mu \int_{0}^{t}\left\|\boldsymbol{B}^{n}\right\|_{1,2}^{2} d s \leq\left\|\boldsymbol{v}_{0}\right\|_{2}^{2}+\left\|\boldsymbol{B}_{0}\right\|_{2}^{2}
$$

The above inequality implies that the existence time is independent of $n$ and it is possible to take $T=T^{n}$.

As a consequence from (4.7) and Lemma 2.1, we derive that there exists $C>0$ such that

$$
\begin{array}{r}
\left\|D_{N, \theta}\left(\boldsymbol{w}^{n}\right)\right\|_{L^{\infty}\left(0, T ; \boldsymbol{H}_{\sigma}^{\theta}\right)}+\left\|\boldsymbol{w}^{n}\right\|_{L^{\infty}\left(0, T ; \boldsymbol{H}_{\sigma}^{\theta}\right)}+\left\|\boldsymbol{B}^{n}\right\|_{L^{\infty}\left(0, T ; \boldsymbol{L}_{\sigma}^{2}\right)} \leq C \\
\left\|\boldsymbol{B}^{n}\right\|_{L^{2}\left(0, T ; \boldsymbol{H}_{\sigma}^{1}\right)} \leq C
\end{array}
$$

From (4.8), and by using Hölder inequality combined with Sobolev injection we get

$$
\boldsymbol{B}^{n} \otimes \boldsymbol{B}^{n} \in L^{4}\left(0, T ; H^{-1}\left(\mathbb{T}_{3}\right)^{3 \times 3}\right)
$$

Furthermore, (4.8) combined with Lemma 2.3-(ii), Lemma 2.3-(iii), and Lemma 2.3(iv) gives

$$
\begin{array}{r}
D_{N, \theta}\left(\boldsymbol{w}^{n}\right) \otimes D_{N, \theta}\left(\boldsymbol{w}^{n}\right) \in L^{\infty}\left(0, T ; H^{2 \theta-\frac{3}{2}}\left(\mathbb{T}_{3}\right)^{3 \times 3}\right), \\
D_{N, \theta}\left(\boldsymbol{w}^{n}\right) \otimes \boldsymbol{B}^{n} \in L^{2}\left(0, T ; H^{\theta-\frac{1}{2}}\left(\mathbb{T}_{3}\right)^{3 \times 3}\right), \\
\boldsymbol{B}^{n} \otimes D_{N, \theta}\left(\boldsymbol{w}^{n}\right) \in L^{2}\left(0, T ; H^{\theta-\frac{1}{2}}\left(\mathbb{T}_{3}\right)^{3 \times 3}\right), \\
\boldsymbol{B}^{n} \otimes \boldsymbol{B}^{n} \in L^{\frac{6}{5}}\left(0, T ; H^{\frac{1}{6}}\left(\mathbb{T}_{3}\right)^{3 \times 3}\right) .
\end{array}
$$

Note that $L^{2}\left(0, T ; H^{\theta-\frac{1}{2}}\left(\mathbb{T}_{3}\right)^{3 \times 3}\right) \hookrightarrow L^{2}\left(0, T ; L^{2}\left(\mathbb{T}_{3}\right)^{3 \times 3}\right)$ when $\theta \geq \frac{1}{2}$. Thus,

$$
\begin{aligned}
& D_{N, \theta}\left(\boldsymbol{w}^{n}\right) \otimes \boldsymbol{B}^{n} \in L^{2}\left(0, T ; L^{2}\left(\mathbb{T}_{3}\right)^{3 \times 3}\right), \\
& \boldsymbol{B}^{n} \otimes D_{N, \theta}\left(\boldsymbol{w}^{n}\right) \in L^{2}\left(0, T ; L^{2}\left(\mathbb{T}_{3}\right)^{3 \times 3}\right) .
\end{aligned}
$$

From (4.9), (4.10) and (2.2) it follows that, for any $\theta \geq \frac{5}{6}$

$$
\begin{aligned}
\overline{D_{N, \theta}\left(\boldsymbol{w}^{n}\right) \otimes D_{N, \theta}\left(\boldsymbol{w}^{n}\right)} & \in L^{\infty}\left(0, T ; H^{4 \theta-\frac{3}{2}}\right) \\
& \hookrightarrow L^{4}\left(0, T ; H^{2 \theta-1}\left(\mathbb{T}_{3}\right)^{3 \times 3}\right) \cap L^{\frac{6}{5}}\left(0, T ; H^{2 \theta+\frac{1}{6}}\left(\mathbb{T}_{3}\right)^{3 \times 3}\right), \\
\overline{\boldsymbol{B}^{n} \otimes \boldsymbol{B}^{n}} & \in L^{4}\left(0, T ; H^{2 \theta-1}\left(\mathbb{T}_{3}\right)^{3 \times 3}\right) \cap L^{\frac{6}{5}}\left(0, T ; H^{2 \theta+\frac{1}{6}}\left(\mathbb{T}_{3}\right)^{3 \times 3}\right) .
\end{aligned}
$$

As in the proof of Theorem 1.1 and by using (4.8)-(4.12), we can derive the following 
estimates

$$
\begin{array}{r}
\int_{0}^{T}\left\|q^{n}\right\|_{2 \theta-1,2}^{4} d t<C, \\
\int_{0}^{T}\left\|\partial_{t} \boldsymbol{w}^{n}\right\|_{2 \theta-2,2}^{4} d t<C \text { and } \int_{0}^{T}\left\|\partial_{t} \boldsymbol{w}^{n}\right\|_{2 \theta-\frac{5}{6}}^{\frac{6}{5}} d t<C, \\
\int_{0}^{T}\left\|\partial_{t} \boldsymbol{B}^{n}\right\|_{-1,2}^{2} d t<C .
\end{array}
$$

Step 1-iii (Limit $n \rightarrow \infty$ part I). It follows from the estimates (4.8)-(4.13) and the Aubin-Lions compactness lemma (see [14] for example) that there are a not relabeled subsequence of $\left(\boldsymbol{w}^{n}, \boldsymbol{B}^{n}, q^{n},\right)$ and a triplet $(\boldsymbol{w}, \boldsymbol{B}, q)$ such that

$$
\begin{aligned}
& \boldsymbol{w}^{n} \rightarrow^{*} \boldsymbol{w} \text { and } D_{N, \theta}\left(\boldsymbol{w}^{n}\right) \rightarrow^{*} D_{N, \theta}(\boldsymbol{w}) \quad \text { weakly* in } L^{\infty}\left(0, T ; \boldsymbol{H}_{\sigma}^{\theta}\right) \text {, } \\
& \boldsymbol{B}^{n} \boldsymbol{-}^{*} \boldsymbol{B} \quad \text { weakly* in } L^{\infty}\left(0, T ; \boldsymbol{L}_{\sigma}^{2}\right), \\
& \boldsymbol{B}^{n} \rightarrow \boldsymbol{B} \quad \text { weakly in } L^{2}\left(0, T ; \boldsymbol{H}_{\sigma}^{1}\right), \\
& \partial_{t} \boldsymbol{w}^{n} \rightarrow \partial_{t} \boldsymbol{w} \quad \text { weakly in } L^{4}\left(0, T ; \boldsymbol{H}^{2 \theta-2}\right) \cap L^{\frac{6}{5}}\left(0, T ; \boldsymbol{H}^{2 \theta-\frac{5}{6}}\right) \text {, } \\
& \partial_{t} \boldsymbol{B}^{n} \rightarrow \partial_{t} \boldsymbol{B} \quad \text { weakly in } L^{2}\left(0, T ; \boldsymbol{H}^{-1}\right), \\
& q^{n} \rightarrow q \quad \text { weakly in } L^{4}\left(0, T ; H^{2 \theta-1}\left(\mathbb{T}_{3}\right)\right), \\
& \boldsymbol{w}^{n} \rightarrow \boldsymbol{w} \text { and } D_{N, \theta}\left(\boldsymbol{w}^{n}\right) \rightarrow D_{N, \theta}(\boldsymbol{w}) \quad \text { strongly in } L^{2}\left(0, T ; \boldsymbol{L}_{\sigma}^{2}\right) \text {, } \\
& \boldsymbol{B}^{n} \rightarrow \boldsymbol{B} \quad \text { strongly in } L^{2}\left(0, T ; \boldsymbol{L}_{\sigma}^{2}\right) .
\end{aligned}
$$

From (4.20) it follows that

$$
\overline{D_{N, \theta}\left(\boldsymbol{w}^{n}\right) \otimes D_{N, \theta}\left(\boldsymbol{w}^{n}\right)} \rightarrow \overline{D_{N, \theta}(\boldsymbol{w}) \otimes D_{N, \theta}(\boldsymbol{w})} \text { strongly in } L^{1}\left(0, T ; L^{1}\left(\mathbb{T}_{3}\right)^{3 \times 3}\right) .
$$

From (4.16) and (4.20) it follows that

$$
\begin{array}{ll}
\boldsymbol{B}^{n} \otimes D_{N, \theta}\left(\boldsymbol{w}^{n}\right) \rightarrow \boldsymbol{B} \otimes D_{N, \theta}(\boldsymbol{w}) & \text { strongly in } L^{1}\left(0, T ; L^{1}\left(\mathbb{T}_{3}\right)^{3 \times 3}\right), \\
D_{N, \theta}\left(\boldsymbol{w}^{n}\right) \otimes \boldsymbol{B}^{n} \rightarrow D_{N, \theta}(\boldsymbol{w}) \otimes \boldsymbol{B} & \text { strongly in } L^{1}\left(0, T ; L^{1}\left(\mathbb{T}_{3}\right)^{3 \times 3}\right) .
\end{array}
$$

From (4.16) and (4.21) it follows that

$$
\overline{\boldsymbol{B}^{n} \otimes \boldsymbol{B}^{n}} \rightarrow \overline{\boldsymbol{B} \otimes \boldsymbol{B}} \quad \text { strongly in } L^{1}\left(0, T ; L^{1}\left(\mathbb{T}_{3}\right)^{3 \times 3}\right) .
$$

Since the sequence $\left\{\overline{D_{N, \theta}\left(\boldsymbol{w}^{n}\right) \otimes D_{N, \theta}\left(\boldsymbol{w}^{n}\right)}\right\}_{n \in \mathbb{N}} \quad$ is bounded in $L^{4}\left(0, T ; H^{2 \theta-1}\left(\mathbb{T}_{3}\right)^{3 \times 3}\right)$, it converges weakly, up to a subsequence, to some $\psi$ in $L^{4}\left(0, T ; H^{2 \theta-1}\left(\mathbb{T}_{3}\right)^{3 \times 3}\right)$. The result above and uniqueness of the limit, allows us to claim that $\psi=\overline{D_{N, \theta}(\boldsymbol{w}) \otimes D_{N, \theta}(\boldsymbol{w})}$. Consequently

$\overline{D_{N, \theta}\left(\boldsymbol{w}^{n}\right) \otimes D_{N, \theta}\left(\boldsymbol{w}^{n}\right)}-\overline{D_{N, \theta}(\boldsymbol{w}) \otimes D_{N, \theta}(\boldsymbol{w})} \quad$ weakly in $L^{4}\left(0, T ; H^{2 \theta-1}\left(\mathbb{T}_{3}\right)^{3 \times 3}\right)$.

We also observe that

$$
\begin{aligned}
& D_{N, \theta}\left(\boldsymbol{w}^{n}\right) \otimes \boldsymbol{B}^{n} \rightarrow D_{N, \theta}(\boldsymbol{w}) \otimes \boldsymbol{B} \quad \text { weakly in } L^{2}\left(0, T ; L^{2}\left(\mathbb{T}_{3}\right)^{3 \times 3}\right), \\
& \boldsymbol{B}^{n} \otimes D_{N, \theta}\left(\boldsymbol{w}^{n}\right) \rightarrow \boldsymbol{B} \otimes D_{N, \theta}(\boldsymbol{w}) \quad \text { weakly in } L^{2}\left(0, T ; L^{2}\left(\mathbb{T}_{3}\right)^{3 \times 3}\right) \text {, } \\
& \overline{\boldsymbol{B}^{n} \otimes \boldsymbol{B}^{n}} \rightarrow \overline{\boldsymbol{B} \otimes \boldsymbol{B}} \quad \text { weakly in } L^{4}\left(0, T ; H^{2 \theta-1}\left(\mathbb{T}_{3}\right)^{3 \times 3}\right) \text {. }
\end{aligned}
$$


The above established convergences are clearly sufficient for taking the limit in (4.2)(4.3) and for concluding that $\boldsymbol{w}, \boldsymbol{B}$ and $q$ satisfy (1.15)-(1.16). Moreover, from (4.14) and (4.17) and by using that $\theta \geq \frac{5}{6}$, we obtain that $\boldsymbol{w} \in L^{2}\left(0, T ; \boldsymbol{H}_{\sigma}^{\theta}\right)$ and $\partial_{t} \boldsymbol{w} \in L^{2}\left(0, T ; \boldsymbol{H}^{-\theta}\right)$. Thus, we deduce by a classical argument ([13]) that

$$
\boldsymbol{w} \in \mathcal{C}\left(0, T ; \boldsymbol{L}_{\sigma}^{2}\right)
$$

Similarly, we deduce from (4.16) and (4.18) that

$$
\boldsymbol{B} \in \mathcal{C}\left(0, T ; \boldsymbol{L}_{\sigma}^{2}\right) .
$$

Furthermore, from the strong continuity of $\boldsymbol{w}$ and $\boldsymbol{B}$ with respect to the time with value in $\boldsymbol{L}_{\sigma}^{2}$, we deduce that $\boldsymbol{w}(0)=\boldsymbol{w}_{0}$ and $\boldsymbol{B}(0)=\boldsymbol{B}_{0}$.

As we have seen, $\partial_{t} \boldsymbol{w} \in L^{\frac{6}{5}}\left(0, T ; \boldsymbol{H}^{2 \theta-\frac{5}{6}}\right)$. Thus, we deduce that

$$
\partial_{t} \boldsymbol{w} \in L^{\frac{6}{5}}\left(0, T ; \boldsymbol{H}^{\theta}\right) \text { for any } \theta \geq \frac{5}{6} .
$$

Therefore, we get by using the following identity

$$
\boldsymbol{w}(t, \cdot)=\boldsymbol{w}\left(t^{\prime}, \cdot\right)+\int_{t^{\prime}}^{t} \partial_{s} \boldsymbol{w} d s
$$

that

$$
\boldsymbol{w} \in \mathcal{C}\left(0, T ; \boldsymbol{H}_{\sigma}^{\theta}\right) \text { for almost all } t \in[0, T] .
$$

Step 2 (Existence of regular weak solutions). Now, we show the existence of regular weak solutions, assuming in addition that $\boldsymbol{B}_{0} \in \boldsymbol{H}_{\sigma}^{1}$.

Step 2-i (New uniform estimates via a regularity approach). Multiplying the $r$ th equation in (4.2) with $\alpha^{2 \theta}|\boldsymbol{k}|^{4 \theta} \widehat{D_{N, \theta}} \boldsymbol{c}_{r}^{n}(t)+|\boldsymbol{k}|^{2 \theta} \widehat{D_{N, \theta}} \boldsymbol{c}_{r}^{n}(t)$, and multiplying the $r$ th equation in (4.3) with $|\boldsymbol{k}|^{2} \boldsymbol{d}_{r}^{n}(t)$, summing the resulted equations over $r=1,2, \ldots, n$, (in short, we use $\mathbb{A}_{\theta}(-\Delta)^{\theta} D_{N, \theta}\left(\boldsymbol{w}^{n}\right)$ as a test function in (4.2) and $-\Delta \boldsymbol{B}^{n}$ as a test function in (4.3)), and integrating by parts, we come to the following inequality

$$
\begin{aligned}
& \frac{1}{2} \frac{d}{d t}\left(\left\|\mathbb{A}_{\theta}^{\frac{1}{2}} D_{N, \theta}^{\frac{1}{2}}\left(\boldsymbol{w}^{n}\right)\right\|_{\theta, 2}^{2}+\left\|\nabla \boldsymbol{B}^{n}\right\|_{2}^{2}\right)+\mu\left\|\nabla \boldsymbol{B}^{n}\right\|_{1,2}^{2} \\
& \leq\left|\int_{\mathbb{T}_{3}}\left(D_{N, \theta}\left(\boldsymbol{w}^{n}\right) \cdot \nabla\right) \boldsymbol{B}^{n} \Delta \boldsymbol{B}^{n}\right| d \boldsymbol{x}+\left|\int_{\mathbb{T}_{3}}\left(D_{N, \theta}\left(\boldsymbol{w}^{n}\right) \cdot \nabla\right) D_{N, \theta}\left(\boldsymbol{w}^{n}\right)(-\Delta)^{\theta} D_{N, \theta}\left(\boldsymbol{w}^{n}\right)\right| d \boldsymbol{x} \\
& \quad+\left|\int_{\mathbb{T}_{3}}\left(\boldsymbol{B}^{n} \cdot \nabla\right) \boldsymbol{B}^{n}(-\Delta)^{\theta} D_{N, \theta}\left(\boldsymbol{w}^{n}\right)\right| d \boldsymbol{x}+\left|\int_{\mathbb{T}_{3}}\left(\boldsymbol{B}^{n} \cdot \nabla\right) D_{N, \theta}\left(\boldsymbol{w}^{n}\right) \Delta \boldsymbol{B}^{n}\right| d \boldsymbol{x} .
\end{aligned}
$$

The first term in right hand side is estimated by

$$
\begin{aligned}
\left|\int_{\mathbb{T}_{3}}\left(D_{N, \theta}\left(\boldsymbol{w}^{n}\right) \cdot \nabla\right) \boldsymbol{B}^{n} \Delta \boldsymbol{B}^{n}\right| d \boldsymbol{x} & \leq C\left\|D_{N, \theta}\left(\boldsymbol{w}^{n}\right)\right\|_{\theta, 2}\left\|\nabla \boldsymbol{B}^{n}\right\|_{\frac{3}{2}-\theta, 2}\left\|\Delta \boldsymbol{B}^{n}\right\|_{2} \\
& \leq C\left\|D_{N, \theta}\left(\boldsymbol{w}^{n}\right)\right\|_{\theta, 2}\left\|\nabla \boldsymbol{B}^{n}\right\|_{2}^{\theta-\frac{1}{2}}\left\|\Delta \boldsymbol{B}^{n}\right\|_{2}^{\frac{5}{2}-\theta} .
\end{aligned}
$$

Where we have used Lemma 2.2, together with the Cauchy-Schwarz inequality and the following interpolation inequality

$$
\left\|\nabla \boldsymbol{B}^{n}\right\|_{\frac{3}{2}-\theta, 2} \leq\left\|\nabla \boldsymbol{B}^{n}\right\|_{2}^{\theta-\frac{1}{2}}\left\|\nabla \boldsymbol{B}^{n}\right\|_{1,2}^{\frac{3}{2}-\theta}
$$


Similarly, we can estimate the second term in the right hand side by

$$
\begin{aligned}
& \left|\int_{\mathbb{T}_{3}}\left(D_{N, \theta}\left(\boldsymbol{w}^{n}\right) \cdot \nabla\right) D_{N, \theta}\left(\boldsymbol{w}^{n}\right)(-\Delta)^{\theta} D_{N, \theta}\left(\boldsymbol{w}^{n}\right)\right| d \boldsymbol{x} \\
& \leq C\left\|D_{N, \theta}\left(\boldsymbol{w}^{n}\right)\right\|_{\theta, 2}\left\|D_{N, \theta}\left(\boldsymbol{w}^{n}\right)\right\|_{\frac{5}{2}-2 \theta, 2}\left\|D_{N, \theta}\left(\boldsymbol{w}^{n}\right)\right\|_{2 \theta, 2} .
\end{aligned}
$$

Since $\theta \geq \frac{5}{6}$, we have $\frac{5}{2}-2 \theta \leq \theta$ and $\left\|D_{N, \theta}\left(\boldsymbol{w}^{n}\right)\right\|_{\frac{5}{2}-2 \theta} \leq C\left\|D_{N, \theta}\left(\boldsymbol{w}^{n}\right)\right\|_{\theta, 2}$. Thus, we get

$$
\begin{aligned}
& \left|\int_{\mathbb{T}_{3}}\left(D_{N, \theta}\left(\boldsymbol{w}^{n}\right) \cdot \nabla\right) D_{N, \theta}\left(\boldsymbol{w}^{n}\right)(-\Delta)^{\theta} D_{N, \theta}\left(\boldsymbol{w}^{n}\right)\right| d \boldsymbol{x} \\
& \leq C\left\|D_{N, \theta}\left(\boldsymbol{w}^{n}\right)\right\|_{\theta, 2}\left\|D_{N, \theta}\left(\boldsymbol{w}^{n}\right)\right\|_{2 \theta, 2}^{2} .
\end{aligned}
$$

The third term in the right hand side is estimated by

$$
\left|\int_{\mathbb{T}_{3}}\left(\boldsymbol{B}^{n} \cdot \nabla\right) \boldsymbol{B}^{n}(-\Delta)^{\theta} D_{N, \theta}\left(\boldsymbol{w}^{n}\right)\right| d \boldsymbol{x} \leq C\left\|\boldsymbol{B}^{n}\right\|_{1,2}\left\|\boldsymbol{B}^{n}\right\|_{2,2}\left\|D_{N, \theta}\left(\boldsymbol{w}^{n}\right)\right\|_{2 \theta, 2} .
$$

The last term in the right hand side is estimated by

$$
\left|\int_{\mathbb{T}_{3}}\left(\boldsymbol{B}^{n} \cdot \nabla\right) D_{N, \theta}\left(\boldsymbol{w}^{n}\right) \Delta \boldsymbol{B}^{n}\right| d \boldsymbol{x} \leq C\left\|\boldsymbol{B}^{n}\right\|_{1,2}\left\|D_{N, \theta}\left(\boldsymbol{w}^{n}\right)\right\|_{2 \theta, 2}\left\|\Delta \boldsymbol{B}^{n}\right\|_{2},
$$

where we have used once more Lemma 2.2 , and the fact that $\left\|\boldsymbol{B}^{n}\right\|_{\frac{5}{2}-2 \theta, 2} \leq C\left\|\boldsymbol{B}^{n}\right\|_{1,2}$ for all $\theta \geq \frac{5}{6}$.

Therefore,

$$
\begin{aligned}
& \frac{1}{2} \frac{d}{d t}\left(\left\|\mathbb{A}_{\theta}^{\frac{1}{2}} D_{N, \theta}^{\frac{1}{2}}\left(\boldsymbol{w}^{n}\right)\right\|_{\theta, 2}^{2}+\left\|\nabla \boldsymbol{B}^{n}\right\|_{2}^{2}\right)+\mu\left\|\nabla \boldsymbol{B}^{n}\right\|_{1,2}^{2} \\
& \leq C\left\|D_{N, \theta}\left(\boldsymbol{w}^{n}\right)\right\|_{\theta, 2}\left\|\nabla \boldsymbol{B}^{n}\right\|_{2}^{\theta-\frac{1}{2}} C\left\|\Delta \boldsymbol{B}^{n}\right\|_{2}^{\frac{5}{2}-\theta}+\left\|D_{N, \theta}\left(\boldsymbol{w}^{n}\right)\right\|_{\theta, 2}\left\|D_{N, \theta}\left(\boldsymbol{w}^{n}\right)\right\|_{2 \theta, 2}^{2} \\
& \quad+2 C\left\|\boldsymbol{B}^{n}\right\|_{1,2}\left\|D_{N, \theta}\left(\boldsymbol{w}^{n}\right)\right\|_{2 \theta, 2}\left\|\Delta \boldsymbol{B}^{n}\right\|_{2} .
\end{aligned}
$$

Thus, by using the Young inequality combined with Lemma 2.1 we get

$$
\begin{aligned}
& \frac{d}{d t}\left(\alpha^{2 \theta}\left\|\boldsymbol{w}^{n}\right\|_{2 \theta, 2}^{2}+\left\|\boldsymbol{B}^{n}\right\|_{1,2}^{2}\right)+\mu\left\|\nabla \boldsymbol{B}^{n}\right\|_{1,2}^{2} \\
& \leq C(N+1)^{5}\left\|\boldsymbol{w}^{n}\right\|_{\theta, 2}^{\frac{4}{2 \theta-1}}\left\|\nabla \boldsymbol{B}^{n}\right\|_{2}^{2}+C(N+1)^{3}\left\|\boldsymbol{w}^{n}\right\|_{\theta, 2}\left\|\boldsymbol{w}^{n}\right\|_{2 \theta, 2}^{2} \\
& \quad+C(N+1)^{2}\left\|\boldsymbol{B}^{n}\right\|_{1,2}^{2}\left\|\boldsymbol{w}^{n}\right\|_{2 \theta, 2}^{2}
\end{aligned}
$$

Therefore,

$$
\begin{aligned}
& \frac{d}{d t}\left(\alpha^{2 \theta}\left\|\boldsymbol{w}^{n}\right\|_{2 \theta, 2}^{2}+\left\|\boldsymbol{B}^{n}\right\|_{1,2}^{2}\right)+\mu\left\|\nabla \boldsymbol{B}^{n}\right\|_{1,2}^{2} \\
& \quad \leq C(t)(N+1)^{5}\left(\left\|\boldsymbol{w}^{n}\right\|_{2 \theta, 2}^{2}+\left\|\boldsymbol{B}^{n}\right\|_{1,2}^{2}\right),
\end{aligned}
$$

where $C(t)=\left(\left\|\boldsymbol{w}^{n}\right\|_{\theta, 2}^{\frac{4}{2 \theta-1}}+\left\|\boldsymbol{w}^{n}\right\|_{\theta, 2}+\left\|\boldsymbol{B}^{n}\right\|_{1,2}^{2}\right) \in L^{1}(0, T)$.

Thus, by using Gronwall's lemma we obtain

$$
\sup _{t \in[0, T]}\left(\alpha^{2 \theta}\left\|\boldsymbol{w}^{n}\right\|_{2 \theta, 2}^{2}+\left\|\boldsymbol{B}^{n}\right\|_{1,2}^{2}\right) \leq C\left(\alpha^{2 \theta}, N\right)\left(\left\|\boldsymbol{w}_{0}\right\|_{2 \theta, 2}^{2}+\left\|\boldsymbol{B}_{0}\right\|_{1,2}^{2}\right) \exp \int_{0}^{T} C(t) d t .
$$


Integrate $(4.35)$ on $[0, T]$ we get

$$
\sup _{t \in[0, T]}\left(\alpha^{2 \theta}\left\|\boldsymbol{w}^{n}\right\|_{2 \theta, 2}^{2}+\left\|\boldsymbol{B}^{n}\right\|_{1,2}^{2}\right)+\mu \int_{0}^{T}\left\|\nabla \boldsymbol{B}^{n}\right\|_{1,2}^{2} d t \leq C\left(\alpha^{2 \theta}, N, \boldsymbol{w}_{0}, \boldsymbol{B}_{0}, T\right),
$$

where $C\left(\alpha^{2 \theta}, N, \boldsymbol{w}_{0}, \boldsymbol{B}_{0}, T\right)$ does not depend on $n$.

As a consequence from (4.37), we derive that there exists $C>0$ such that

$$
\begin{aligned}
\left\|D_{N, \theta}\left(\boldsymbol{w}^{n}\right)\right\|_{L^{\infty}\left(0, T ; \boldsymbol{H}_{\sigma}^{2 \theta}\right)}+\left\|\boldsymbol{w}^{n}\right\|_{L^{\infty}\left(0, T ; \boldsymbol{H}_{\sigma}^{2 \theta}\right)}+\left\|\boldsymbol{B}^{n}\right\|_{L^{\infty}\left(0, T ; \boldsymbol{H}_{\sigma}^{1}\right)} \leq C, \\
\left\|\boldsymbol{B}^{n}\right\|_{L^{2}\left(0, T ; \boldsymbol{H}_{\sigma}^{2}\right)} \leq C .
\end{aligned}
$$

As in the proof of Theorem 1.1 and by using (4.38), we can derive the following estimates

$$
\begin{array}{r}
\int_{0}^{T}\left\|q^{n}\right\|_{2 \theta+\frac{3}{2}, 2}^{2} d t<C, \\
\int_{0}^{T}\left\|\partial_{t} \boldsymbol{w}^{n}\right\|_{2 \theta+\frac{1}{2}, 2}^{2} d t<C, \\
\int_{0}^{T}\left\|\partial_{t} \boldsymbol{B}^{n}\right\|_{2}^{2} d t<C .
\end{array}
$$

Step 2-ii (Limit $n \rightarrow \infty$ part II). From Step 1-iii, we already know that there exists a weak solution $(\boldsymbol{w}, \boldsymbol{B}, q)$ of (1.15)-(1.16) and not relabeled subsequence of $\left(\boldsymbol{w}^{n}, \boldsymbol{B}^{n}, q^{n}\right)$ such that

$$
\begin{aligned}
\boldsymbol{w}^{n} \rightarrow \boldsymbol{w} \text { and } D_{N, \theta}\left(\boldsymbol{w}^{n}\right) & \rightarrow D_{N, \theta}(\boldsymbol{w}) & & \text { strongly in } L^{2}\left(0, T ; \boldsymbol{L}_{\sigma}^{2}\right), \\
\boldsymbol{B}^{n} & \rightarrow \boldsymbol{B} & & \text { strongly in } L^{2}\left(0, T ; \boldsymbol{L}_{\sigma}^{2}\right), \\
q^{n} & \rightarrow q & & \text { weakly in } L^{2}\left(0, T ; H^{2 \theta-1}\left(\mathbb{T}_{3}\right)\right) .
\end{aligned}
$$

Thus, it follows from the estimates (4.38)-(4.39), the Aubin-Lions compactness lemma (see [14] for example) and the uniqueness of the limit that there are a not relabeled subsequence of $\left(\boldsymbol{w}^{n}, \boldsymbol{B}^{n}, q^{n}\right)$ and a triplet $(\boldsymbol{w}, \boldsymbol{B}, q)$ such that

$$
\begin{aligned}
\boldsymbol{w}^{n} \rightarrow \boldsymbol{w} \text { and } D_{N, \theta}\left(\boldsymbol{w}^{n}\right) & \rightarrow D_{N, \theta}(\boldsymbol{w}) & & \text { strongly in } L^{2}\left(0, T ; \boldsymbol{H}_{\sigma}^{\theta}\right), \\
\boldsymbol{B}^{n} & \rightarrow \boldsymbol{B} & & \text { strongly in } L^{2}\left(0, T ; \boldsymbol{H}_{\sigma}^{1}\right), \\
q^{n} & \rightarrow q & & \text { weakly in } L^{2}\left(0, T ; H^{2 \theta+\frac{3}{2}}\left(\mathbb{T}_{3}\right)\right) .
\end{aligned}
$$

Moreover, we obtain that $\boldsymbol{B} \in L^{2}\left(0, T ; \boldsymbol{H}_{\sigma}^{2}\right)$ and $\partial_{t} \boldsymbol{B} \in L^{2}\left(0, T ; \boldsymbol{L}^{2}\right)$. Thus, we deduce by a classical argument ([13]) that

$$
\boldsymbol{B} \in \mathcal{C}\left(0, T ; \boldsymbol{H}_{\sigma}^{1}\right)
$$

Step 3 (Uniqueness of regular weak solutions). Next, we will prove the uniqueness of regular weak solutions among the class of weak solutions.

Let $\theta \geq \frac{5}{6}$ and $\left(\boldsymbol{w}_{1}, \boldsymbol{B}_{1}, q_{1}\right)$ be a weak solution of (1.1) on the interval $[0, T]$, with initial values $\boldsymbol{v}_{1}(0) \in \boldsymbol{L}_{\sigma}^{2}, \boldsymbol{B}_{1}(0) \in \boldsymbol{H}_{\sigma}^{1}$. Let also $\left(\boldsymbol{w}_{2}, \boldsymbol{B}_{2}, q_{2}\right)$ be a regular weak solution of (1.1) on the interval $[0, T]$, with initial values $\boldsymbol{v}_{2}(0) \in \boldsymbol{L}_{\sigma}^{2}, \boldsymbol{B}_{2}(0) \in \boldsymbol{H}_{\sigma}^{1}$. 
We denote by $\delta \boldsymbol{w}=\boldsymbol{w}_{2}-\boldsymbol{w}_{1}$, by $\delta \boldsymbol{B}=\boldsymbol{B}_{2}-\boldsymbol{B}_{1}$ and by $\delta q=q_{2}-q_{1}$.

Then one has

$$
\begin{array}{r}
\left.\partial_{t} \delta \boldsymbol{w}+\nabla \cdot\left(\overline{D_{N, \theta}\left(\boldsymbol{w}_{2}\right) \otimes D_{N, \theta}\left(\boldsymbol{w}_{2}\right)}\right)-\nabla \cdot\left(\overline{D_{N, \theta}\left(\boldsymbol{w}_{1}\right) \otimes D_{N, \theta}\left(\boldsymbol{w}_{1}\right.}\right)\right) \\
-\nabla \cdot\left(\overline{\boldsymbol{B}_{2} \otimes \boldsymbol{B}_{2}}\right)+\nabla \cdot\left(\overline{\boldsymbol{B}_{1} \otimes \boldsymbol{B}_{1}}\right)+\nabla \delta q=0 \\
\partial_{t} \delta \boldsymbol{B}-\mu \Delta \delta \boldsymbol{B}+\nabla \cdot\left(D_{N, \theta}\left(\boldsymbol{w}_{2}\right) \otimes \boldsymbol{B}_{2}\right)-\nabla \cdot\left(D_{N, \theta}\left(\boldsymbol{w}_{1}\right) \otimes \boldsymbol{B}_{1}\right) \\
-\nabla \cdot\left(\boldsymbol{B}_{2} \otimes D_{N, \theta}\left(\boldsymbol{w}_{2}\right)\right)+\nabla \cdot\left(\boldsymbol{B}_{1} \otimes D_{N, \theta}\left(\boldsymbol{w}_{1}\right)\right)=0
\end{array}
$$

and $\delta \boldsymbol{w}=0, \delta \boldsymbol{B}=0$ at initial time.

Applying $\mathbb{A}_{\theta}^{\frac{1}{2}}$ to the first equation of (4.40) we obtain

$$
\begin{array}{r}
\left.\mathbb{A}_{\theta}^{\frac{1}{2}} \partial_{t} \delta \boldsymbol{w}+\mathbb{A}_{\theta}^{\frac{1}{2}} \nabla \cdot\left(\overline{D_{N, \theta}\left(\boldsymbol{w}_{2}\right) \otimes D_{N, \theta}\left(\boldsymbol{w}_{2}\right)}\right)-\mathbb{A}_{\theta}^{\frac{1}{2}} \nabla \cdot\left(\overline{D_{N, \theta}\left(\boldsymbol{w}_{1}\right) \otimes D_{N, \theta}\left(\boldsymbol{w}_{1}\right.}\right)\right) \\
-\mathbb{A}_{\theta}^{\frac{1}{2}} \nabla \cdot\left(\overline{\boldsymbol{B}_{2} \otimes \boldsymbol{B}_{2}}\right)+\mathbb{A}_{\theta}^{\frac{1}{2}} \nabla \cdot\left(\overline{\boldsymbol{B}_{1} \otimes \boldsymbol{B}_{1}}\right)+\mathbb{A}_{\theta}^{\frac{1}{2}} \nabla \delta q=0 .
\end{array}
$$

One can take $\mathbb{A}_{\theta}^{\frac{1}{2}} D_{N, \theta}(\delta \boldsymbol{w}) \in \mathcal{C}\left(0, T ; \boldsymbol{L}_{\sigma}^{2}\right)$ as test function in (4.41) and $\delta \boldsymbol{B} \in$ $L^{2}\left(0, T ; \boldsymbol{H}_{\sigma}^{1}\right)$ as test function in the second equations of (4.40). Let us mention that, $\partial_{t} \delta \boldsymbol{w} \in L^{\frac{6}{5}}\left(0, T ; \boldsymbol{H}^{2 \theta-\frac{5}{6}}\right) \hookrightarrow L^{\frac{6}{5}}\left(0, T ; \boldsymbol{H}^{\theta}\right)$ and $\delta \boldsymbol{w} \in \mathcal{C}\left(0, T ; \boldsymbol{H}_{\sigma}^{\theta}\right)$, for any $\theta \geq \frac{5}{6}$, thus Lemma 3.4 in [12] yields to

$$
\left(\partial_{t} \mathbb{A}_{\theta}^{\frac{1}{2}} \delta \boldsymbol{w}, \mathbb{A}_{\theta}^{\frac{1}{2}} D_{N, \theta}(\delta \boldsymbol{w})\right)=\frac{1}{2} \frac{d}{d t}\left\|\mathbb{A}_{\theta}^{\frac{1}{2}} D_{N, \theta}^{\frac{1}{2}}(\delta \boldsymbol{w})\right\|_{2}^{2} .
$$

Since $\partial_{t} \delta \boldsymbol{B} \in L^{2}\left(0, T ; \boldsymbol{H}^{-1}\right)$, by using Lions-Magenes Lemma [13] we may justifiably write

$$
\left\langle\partial_{t} \delta \boldsymbol{B}, \delta \boldsymbol{B}\right\rangle_{\boldsymbol{H}^{-1}, \boldsymbol{H}^{1}}=\frac{1}{2} \frac{d}{d t}\|\delta \boldsymbol{B}\|_{2}^{2} .
$$

Now, we proceed in the exact way as in the proof of theorem 1.1 in order to obtain the following equality

$$
\begin{aligned}
& \frac{1}{2} \frac{d}{d t}\left(\left\|\mathbb{A}^{\frac{1}{2}} D_{N, \theta}^{\frac{1}{2}}(\delta \boldsymbol{w})\right\|_{2}^{2}+\|\delta \boldsymbol{B}\|_{2}^{2}\right)+\mu\|\delta \boldsymbol{B}\|_{1,2}^{2} \\
& =-\int_{\mathbb{T}_{3}}\left(D_{N, \theta}(\delta \boldsymbol{w}) \cdot \nabla\right) D_{N, \theta}\left(\boldsymbol{w}_{2}\right) D_{N, \theta}(\delta \boldsymbol{w})+\int_{\mathbb{T}_{3}} D_{N, \theta}(\delta \boldsymbol{w}) \otimes \boldsymbol{B}_{2}: \nabla \delta \boldsymbol{B} \\
& \quad+\int_{\mathbb{T}_{3}}(\delta \boldsymbol{B} \cdot \nabla) \boldsymbol{B}_{2} D_{N}(\delta \boldsymbol{w})-\int_{\mathbb{T}_{3}} \delta \boldsymbol{B} \otimes D_{N, \theta}\left(\boldsymbol{w}_{2}\right): \nabla \delta \boldsymbol{B} .
\end{aligned}
$$

Next, we estimate the four integrals in the right hand side of (4.42). The estimates are obtained by using Lemma 2.2, the Young inequality and (2.10).

$$
\begin{aligned}
&\left|\int_{\mathbb{T}_{3}}\left(D_{N, \theta}(\delta \boldsymbol{w}) \cdot \nabla\right) D_{N, \theta}\left(\boldsymbol{w}_{2}\right) D_{N, \theta}(\delta \boldsymbol{w})\right| \leq C(N+1)^{6}\|\delta \boldsymbol{w}\|_{\theta, 2}^{2}\left\|\boldsymbol{w}_{2}\right\|_{2 \theta, 2}, \\
&\left|\int_{\mathbb{T}_{3}} D_{N, \theta}(\delta \boldsymbol{w}) \otimes \boldsymbol{B}_{2}: \nabla \delta \boldsymbol{B}\right| \leq \frac{C}{\mu}\left\|D_{N, \theta}(\delta \boldsymbol{w}) \otimes \boldsymbol{B}_{2}\right\|_{2}^{2}+\frac{\mu}{6}\|\nabla \delta \boldsymbol{B}\|_{2}^{2} \\
& \leq \frac{C(N+1)^{2}}{\mu}\|\delta \boldsymbol{w}\|_{\theta, 2}^{2}\left\|\boldsymbol{B}_{2}\right\|_{1,2}^{2}+\frac{\mu}{6}\|\nabla \delta \boldsymbol{B}\|_{2}^{2},
\end{aligned}
$$


DECONVOLUTION MHD WITH FRACTIONAL REGULARIZATION

$$
\begin{aligned}
\left|\int_{\mathbb{T}_{3}}(\delta \boldsymbol{B} \cdot \nabla) \boldsymbol{B}_{2} D_{N}(\delta \boldsymbol{w})\right| & \leq C(N+1)^{2}\|\delta \boldsymbol{B}\|_{1,2}\left\|\boldsymbol{B}_{2}\right\|_{2,2}\|\delta \boldsymbol{w}\|_{\theta, 2} \\
& \leq \frac{C(N+1)^{2}}{\mu}\left\|\boldsymbol{B}_{2}\right\|_{2,2}^{2}\|\delta \boldsymbol{w}\|_{\theta, 2}^{2}+\frac{\mu}{6}\|\delta \boldsymbol{B}\|_{1,2}^{2} \\
\left|\int_{\mathbb{T}_{3}} \delta \boldsymbol{B} \otimes D_{N, \theta}\left(\boldsymbol{w}_{2}\right): \nabla \delta \boldsymbol{B}\right| & \leq \frac{C(N+1)^{2}}{\mu}\|\delta \boldsymbol{B}\|_{2}^{2}\left\|D_{N, \theta}\left(\boldsymbol{w}_{2}\right)\right\|_{2 \theta, 2}^{2}+\frac{\mu}{6}\|\delta \boldsymbol{B}\|_{1,2}^{2} .
\end{aligned}
$$

By using (2.13) we have

$$
\begin{aligned}
& \frac{1}{2} \frac{d}{d t}\left(\alpha^{2 \theta}\|\delta \boldsymbol{w}\|_{\theta, 2}^{2}+\|\delta \boldsymbol{B}\|_{2}^{2}\right)+\mu\|\delta \boldsymbol{B}\|_{1,2}^{2} \\
& \leq \frac{1}{2} \frac{d}{d t}\left(\left\|\mathbb{A}_{\theta}^{\frac{1}{2}} D_{N, \theta}^{\frac{1}{2}}(\delta \boldsymbol{w})\right\|_{2}^{2}+\|\delta \boldsymbol{B}\|_{2}^{2}\right)+\mu\|\delta \boldsymbol{B}\|_{1,2}^{2} .
\end{aligned}
$$

From (4.42)-(4.47) we get

$$
\begin{aligned}
& \frac{d}{d t}\left(\alpha^{2 \theta}\|\delta \boldsymbol{w}\|_{\theta, 2}^{2}+\|\delta \boldsymbol{B}\|_{2}^{2}\right)+\mu\|\delta \boldsymbol{B}\|_{1,2}^{2} \\
& \leq C(N+1)^{6}\|\delta \boldsymbol{w}\|_{\theta, 2}^{2}\left\|\boldsymbol{w}_{2}\right\|_{2 \theta, 2}+\frac{C(N+1)^{2}}{\mu}\|\delta \boldsymbol{w}\|_{\theta, 2}^{2}\left\|\boldsymbol{B}_{2}\right\|_{1,2}^{2} \\
& \quad+\frac{C(N+1)^{2}}{\mu}\|\delta \boldsymbol{w}\|_{\theta, 2}^{2}\left\|\boldsymbol{B}_{2}\right\|_{2,2}^{2}+\frac{C(N+1)^{2}}{\mu}\|\delta \boldsymbol{B}\|_{2}^{2}\left\|\boldsymbol{w}_{2}\right\|_{2 \theta, 2}^{2} .
\end{aligned}
$$

Hence,

$$
\begin{aligned}
& \frac{d}{d t}\left(\alpha^{2 \theta}\|\delta \boldsymbol{w}\|_{\theta, 2}^{2}+\|\delta \boldsymbol{B}\|_{2}^{2}\right)+\mu\|\delta \boldsymbol{B}\|_{1,2}^{2} \\
& \leq \frac{C(N+1)^{6}}{\min \left(\alpha^{2 \theta}, \mu\right)}\left(\alpha^{2 \theta}\|\delta \boldsymbol{w}\|_{\theta, 2}^{2}+\|\delta \boldsymbol{B}\|_{2}^{2}\right)\left(\left\|\boldsymbol{w}_{2}\right\|_{2 \theta, 2}+\left\|\boldsymbol{w}_{2}\right\|_{2 \theta, 2}^{2}+\left\|\boldsymbol{B}_{2}\right\|_{2,2}^{2}\right)
\end{aligned}
$$

Since $\left\|\boldsymbol{w}_{2}\right\|_{2 \theta, 2}+\left\|\boldsymbol{w}_{2}\right\|_{2 \theta, 2}^{2}+\left\|\boldsymbol{B}_{2}\right\|_{1,2}^{2} \in L^{1}([0, T])$, we conclude by using Gronwall's inequality the continuous dependence of the solutions on the initial data. In particular, if $\delta \boldsymbol{w}_{0}=\delta \boldsymbol{B}_{0}=0$ then $\delta \boldsymbol{w}=\delta \boldsymbol{B}=0$ and the solutions are unique for all $t \in[0, T]$. Since $T>0$ is arbitrary this solution may be uniquely extended for all time.

This finishes the proof of Theorem 1.2.

5. Limit when $N \rightarrow \infty$ in the double viscous case . Let $\left(\boldsymbol{w}_{N}, \boldsymbol{B}_{N}, q_{N}\right)$ be the unique solution of (1.1) constructed in theorem (1.1) with $N>0$ fixed and $\frac{1}{2} \leq \theta \leq 1$. The main result of this section is the following.

TheOREM 5.1. Let $\alpha>0$ and $\frac{1}{2} \leq \theta<1$, then from the sequence $\left\{\left(\boldsymbol{w}_{N}, \boldsymbol{B}_{N}, q_{N}\right)\right\}_{N \in \mathbb{N}}$, one can extract a not relabeled subsequence $\left\{\left(\boldsymbol{w}_{N}, \boldsymbol{B}_{N}, q_{N}\right)\right\}_{N \in \mathbb{N}}$ such that when $N$ tends to $\infty$ :

$\left(\boldsymbol{w}_{N}, \boldsymbol{B}_{N}, q_{N}\right) \rightarrow(\boldsymbol{w}, \boldsymbol{B}, q)$ where

$$
\begin{aligned}
(\boldsymbol{w}, \boldsymbol{B}, q) \in L^{\infty}\left([0, T] ; \boldsymbol{H}_{\sigma}^{\theta}\right) & \cap L^{2}\left([0, T] ; \boldsymbol{H}_{\sigma}^{1+\theta}\right) \times L^{\infty}\left([0, T] ; \boldsymbol{L}_{\sigma}^{2}\right) \\
& \cap L^{2}\left([0, T] ; \boldsymbol{H}_{\sigma}^{1}\right) \times L^{2}\left([0, T] ; H^{-\frac{1}{2}+2 \theta}\left(\mathbb{T}_{3}\right)\right)
\end{aligned}
$$


is a distributional solution of the following system with periodic boundary conditions

$$
\left\{\begin{array}{l}
\partial_{t} \boldsymbol{w}+\nabla \cdot\left(\overline{\mathbb{A}_{\theta}(\boldsymbol{w}) \otimes \mathbb{A}_{\theta}(\boldsymbol{w})}\right)-\nabla \cdot(\overline{\boldsymbol{B} \otimes \boldsymbol{B}})-\nu \Delta \boldsymbol{w}+\nabla q=0 \\
\partial_{t} \boldsymbol{B}+\nabla \cdot\left(\boldsymbol{B} \otimes \mathbb{A}_{\theta}(\boldsymbol{w})\right)-\nabla \cdot\left(\mathbb{A}_{\theta}(\boldsymbol{w}) \otimes \boldsymbol{B}\right)-\mu \Delta \boldsymbol{B}=0 \\
\nabla \cdot \boldsymbol{w}=\nabla \cdot \boldsymbol{B}=0, \int_{\mathbb{T}_{3}} \boldsymbol{w}=\int_{\mathbb{T}_{3}} \boldsymbol{B}=0 \\
\boldsymbol{w}_{t=0}=\boldsymbol{w}_{0}=\overline{\boldsymbol{v}_{0}}, \boldsymbol{B}_{t=0}=\boldsymbol{B}_{0}
\end{array}\right.
$$

The sequence $\left\{\boldsymbol{w}_{N}\right\}_{N \in \mathbb{N}}$ converges strongly to $\boldsymbol{w}$ in the space $L^{2}\left([0, T] ; \boldsymbol{H}^{s}\right)$ for all $s<1+\theta$, while the sequence $\left\{\boldsymbol{B}_{N}\right\}_{N \in \mathbb{N}}$ converges strongly to $\boldsymbol{B}$ in the space $L^{2}\left([0, T] ; \boldsymbol{H}^{s}\right)$ for all $s<1$, and $\left\{q_{N}\right\}_{N \in \mathbb{N}}$ converges weakly to $q$ in the space $L^{2}\left([0, T] ; H^{-\frac{1}{2}+2 \theta}\left(\mathbb{T}_{3}\right)\right)$.

REMARK 5.1. The restriction in the case of the Approximate Deconvolution Model (ADM) in [5] is $\theta>\frac{3}{4}$. If we consider the case $\boldsymbol{B}=0$, then equations (1.1) with $\nu>0$ reduce to the $(A D M)$. Thus, the same approach used here can be used to the $A D M$ to get similar results with $\theta \geq \frac{1}{2}$.

Before proving Theorem 5.1, we first record the following Lemma.

Lemma 5.1. Let $\theta>0$ and assume that $\boldsymbol{v} \in L^{2}\left([0, T], \boldsymbol{H}^{2 \theta}\right)$. Then

$$
D_{N, \theta}(\boldsymbol{v}) \rightarrow \mathbb{A}_{\theta}(\boldsymbol{v}) \text { strongly in } L^{2}\left(0, T ; \boldsymbol{L}^{2}\right) \text {, when } N \rightarrow \infty,
$$

and there exist a constant $C$ independent from $N$ such that

$$
\left\|D_{N, \theta}(\boldsymbol{v})\right\|_{2} \leq\left\|\mathbb{A}_{\theta}(\boldsymbol{v})\right\|_{2} \leq C\left(\alpha^{\theta}\right)\|\boldsymbol{v}\|_{2 \theta, 2} .
$$

Proof. The first part of this lemma is given in [5], see also in [3] for an alternative proof. The second part is a direct consequence from the property (2.9) of the operator $D_{N, \theta}$, the relation (2.4) and Poincar inequality.

Proof of Theorem 5.1. The proof of Theorem 5.1 follows the lines of the proof of Theorem 4.1 in [3]. First, we need to reconstruct a uniform estimates for $\left(\boldsymbol{w}_{N}, \boldsymbol{B}_{N}, q_{N}\right)$ with respect to $N$.

Step 1 (Uniform estimates with respect to $N$ ). Following the proof of Theorem 1.1 we obtain that the solution of (1.1) satisfies

$$
\begin{aligned}
& \frac{1}{2}\left(\left\|\mathbb{A}_{\theta}^{\frac{1}{2}} D_{N, \theta}^{\frac{1}{2}}\left(\boldsymbol{w}_{N}\right)\right\|_{2}^{2}+\left\|\boldsymbol{B}_{N}\right\|_{2}^{2}\right)+\int_{0}^{t}\left(\nu\left\|\mathbb{A}_{\theta}^{\frac{1}{2}} D_{N, \theta}^{\frac{1}{2}}\left(\boldsymbol{w}_{N}\right)\right\|_{1,2}^{2}+\mu\left\|\boldsymbol{B}_{N}\right\|_{1,2}^{2}\right) d s \\
& =\frac{1}{2}\left(\left\|\mathbb{A}_{\theta}^{\frac{1}{2}} D_{N, \theta}^{\frac{1}{2}}\left(\overline{\boldsymbol{v}}_{0}\right)\right\|_{2}^{2}+\left\|\boldsymbol{B}_{0}\right\|_{2}^{2}\right)
\end{aligned}
$$

consequently as $\left\|\mathbb{A}_{\theta}^{\frac{1}{2}} D_{N, \theta}^{\frac{1}{2}}\left(\overline{\boldsymbol{v}}_{0}\right)\right\|_{2}^{2} \leq\left\|\boldsymbol{v}_{0}\right\|_{2}^{2}$ we can bound the right hand side by a constant $C$ which is independent from $N$.

We deduce from (5.4) and Lemma 2.1 that

$$
D_{N, \theta}\left(\boldsymbol{w}_{N}\right) \in L^{\infty}\left(0, T ; \boldsymbol{L}_{\sigma}^{2}\right) \cap L^{2}\left(0, T ; \boldsymbol{H}_{\sigma}^{1}\right), \text { uniformly with respect to } N,
$$

and

$$
\begin{array}{r}
\boldsymbol{w}_{N} \in L^{\infty}\left(0, T ; \boldsymbol{H}_{\sigma}^{\theta}\right) \cap L^{2}\left(0, T ; \boldsymbol{H}_{\sigma}^{1+\theta}\right), \text { uniformly with respect to } N, \\
\boldsymbol{B}_{N} \in L^{\infty}\left(0, T ; \boldsymbol{L}_{\sigma}^{2}\right) \cap L^{2}\left(0, T ; \boldsymbol{H}_{\sigma}^{1}\right), \text { uniformly with respect to } N .
\end{array}
$$


We observe from (5.5) that

$$
\begin{aligned}
D_{N, \theta}\left(\boldsymbol{w}_{N}\right) \otimes D_{N, \theta}\left(\boldsymbol{w}_{N}\right) & \in L^{2}\left(0, T ; H^{-\frac{1}{2}}\left(\mathbb{T}_{3}\right)^{3 \times 3}\right), \\
D_{N, \theta}\left(\boldsymbol{w}_{N}\right) \otimes \boldsymbol{B}_{N} & \in L^{2}\left(0, T ; H^{-\frac{1}{2}}\left(\mathbb{T}_{3}\right)^{3 \times 3}\right), \\
\boldsymbol{B}_{N} \otimes D_{N, \theta}\left(\boldsymbol{w}_{N}\right) & \in L^{2}\left(0, T ; H^{-\frac{1}{2}}\left(\mathbb{T}_{3}\right)^{3 \times 3}\right), \\
\boldsymbol{B}_{N} \otimes \boldsymbol{B}_{N} & \in L^{2}\left(0, T ; H^{-\frac{1}{2}}\left(\mathbb{T}_{3}\right)^{3 \times 3}\right) .
\end{aligned}
$$

Thus, from (5.7) and (2.2) we obtain

$$
\begin{array}{r}
\overline{D_{N, \theta}\left(\boldsymbol{w}_{N}\right) \otimes D_{N, \theta}\left(\boldsymbol{w}_{N}\right)} \in L^{2}\left(0, T ; H^{-\frac{1}{2}+2 \theta}\left(\mathbb{T}_{3}\right)^{3}\right), \\
\overline{\boldsymbol{B}_{N} \otimes \boldsymbol{B}_{N}} \in L^{2}\left(0, T ; H^{-\frac{1}{2}+2 \theta}\left(\mathbb{T}_{3}\right)^{3 \times 3}\right) .
\end{array}
$$

For the pressure term $q_{N}$, we deduce that it verifies the following equation

$$
\Delta q_{N}=-\nabla \cdot \nabla \cdot\left(\overline{D_{N, \theta}\left(\boldsymbol{w}_{N}\right) \otimes D_{N, \theta}\left(\boldsymbol{w}_{N}\right)}-\overline{\boldsymbol{B}_{N} \otimes \boldsymbol{B}_{N}}\right),
$$

consequently, the classical elliptic theory combined with (5.8) implies that

$$
\int_{0}^{T}\left\|q_{N}\right\|_{-\frac{1}{2}+2 \theta, 2}^{2} d t<K, \text { uniformly with respect to } N .
$$

From (1.1), (5.7) and (5.8) we also obtain that

$$
\begin{aligned}
\int_{0}^{T}\left\|\partial_{t} \boldsymbol{w}_{N}\right\|_{-\frac{3}{2}+2 \theta, 2}^{2} d t<K, \text { uniformly with respect to } N, \\
\int_{0}^{T}\left\|\partial_{t} \boldsymbol{B}_{N}\right\|_{-\frac{3}{2}, 2}^{2} d t<K, \text { uniformly with respect to } N .
\end{aligned}
$$

Step 2 (Passing to the limit $N \rightarrow \infty$ ). The central issues is how to take the limit in the nonlinear terms $D_{N, \theta}\left(\boldsymbol{w}_{N}\right) \otimes D_{N, \theta}\left(\boldsymbol{w}_{N}\right), \boldsymbol{B}_{N} \otimes D_{N, \theta}\left(\boldsymbol{w}_{N}\right)$ and $D_{N, \theta}\left(\boldsymbol{w}_{N}\right) \otimes \boldsymbol{B}_{N}$. From the Aubin-Lions compactness Lemma (the same arguments as in section 3) we can find a not relabeled subsequence $\left\{\left(\boldsymbol{w}_{N}, D_{N, \theta}\left(\boldsymbol{w}_{N}\right), \boldsymbol{B}_{N}, q_{N}\right)\right\}_{N \in \mathbb{N}}$ and $(\boldsymbol{w}, \boldsymbol{z}, \boldsymbol{B}, q)$ such that when $N \rightarrow \infty$ we have:

$$
\begin{array}{rlrl}
D_{N, \theta}\left(\boldsymbol{w}_{N}\right) & \rightarrow \boldsymbol{z} & & \text { weakly in } L^{2}\left(0, T ; \boldsymbol{H}_{\sigma}^{1}\right), \\
q_{N} & \rightarrow q & \text { weakly in } L^{2}\left(0, T ; H^{-\frac{1}{2}+2 \theta}\left(\mathbb{T}_{3}\right)\right), \\
\boldsymbol{w}_{N} & \rightarrow \boldsymbol{w} & \text { strongly in } L^{2}\left(0, T ; \boldsymbol{H}_{\sigma}^{s}\right) \text { for all } s<1+\theta, \\
\boldsymbol{B}_{N} & \rightarrow \boldsymbol{B} & \text { strongly in } L^{2}\left(0, T ; \boldsymbol{H}_{\sigma}^{s}\right) \text { for all } s<1 .
\end{array}
$$

The goal is to prove

$$
\begin{aligned}
& D_{N, \theta}\left(\boldsymbol{w}_{N}\right) \otimes D_{N, \theta}\left(\boldsymbol{w}_{N}\right) \rightarrow \mathbb{A}_{\theta}(\boldsymbol{w}) \otimes \mathbb{A}_{\theta}(\boldsymbol{w}) \quad \text { strongly in } L^{1}\left(0, T ; L^{1}\left(\mathbb{T}_{3}\right)^{3 \times 3}\right), \\
& D_{N, \theta}\left(\boldsymbol{w}_{N}\right) \otimes \boldsymbol{B}_{N} \rightarrow \mathbb{A}_{\theta}(\boldsymbol{w}) \otimes \boldsymbol{B} \quad \text { strongly in } L^{1}\left(0, T ; L^{1}\left(\mathbb{T}_{3}\right)^{3 \times 3}\right) \text {, } \\
& \boldsymbol{B}_{N} \otimes D_{N, \theta}\left(\boldsymbol{w}_{N}\right) \rightarrow \boldsymbol{B} \otimes \mathbb{A}_{\theta}(\boldsymbol{w}) \quad \text { strongly in } L^{1}\left(0, T ; L^{1}\left(\mathbb{T}_{3}\right)^{3 \times 3}\right) .
\end{aligned}
$$

Thus, it remains to show that

$$
D_{N, \theta}\left(\boldsymbol{w}_{N}\right) \rightarrow \mathbb{A}_{\theta}(\boldsymbol{w}) \quad \text { strongly in } L^{2}\left(0, T ; \boldsymbol{L}^{2}\right) .
$$


In order to show (5.19), we compute directly the difference between $D_{N, \theta}\left(\boldsymbol{w}_{N}\right)$ and $\mathbb{A}_{\theta}(\boldsymbol{w})$ as follows

$$
\begin{aligned}
& \left\|D_{N, \theta}\left(\boldsymbol{w}_{N}\right)-\mathbb{A}_{\theta}(\boldsymbol{w})\right\|_{L^{2}\left(0, T ; L^{2}\right)} \\
& \leq\left\|D_{N, \theta}\left(\boldsymbol{w}_{N}\right)-D_{N, \theta}(\boldsymbol{w})\right\|_{L^{2}\left(0, T ; \boldsymbol{L}^{2}\right)}+\left\|D_{N, \theta}(\boldsymbol{w})-\mathbb{A}_{\theta}(\boldsymbol{w})\right\|_{L^{2}\left(0, T ; \boldsymbol{L}^{2}\right)} \\
& \leq\left\|\mathbb{A}_{\theta}\left(\boldsymbol{w}_{N}-\boldsymbol{w}\right)\right\|_{L^{2}\left(0, T ; \boldsymbol{L}^{2}\right)}+\left\|D_{N, \theta}(\boldsymbol{w})-\mathbb{A}_{\theta}(\boldsymbol{w})\right\|_{L^{2}\left(0, T ; \boldsymbol{L}^{2}\right)} \\
& \leq C\left\|\boldsymbol{w}_{N}-\boldsymbol{w}\right\|_{L^{2}\left(0, T ; \boldsymbol{H}^{2 \theta}\right)}+\left\|D_{N, \theta}(\boldsymbol{w})-\mathbb{A}_{\theta}(\boldsymbol{w})\right\|_{L^{2}\left(0, T ; \boldsymbol{L}^{2}\right)},
\end{aligned}
$$

where we have used the second part of lemma 5.1. Hence, using that $\theta<1$, (5.14) and the first part of lemma (5.1) we deduce (5.19). Finally the result above, (5.19), combined with (5.12) and the uniqueness of the limit, allows us to deduce

$$
D_{N, \theta}\left(\boldsymbol{w}_{N}\right) \rightarrow \mathbb{A}_{\theta}(\boldsymbol{w}) \quad \text { weakly in } L^{2}\left(0, T ; \boldsymbol{H}_{\sigma}^{1}\right) .
$$

Consequently we get (5.16) and by using (5.7) we obtain

$$
\overline{D_{N, \theta}\left(\boldsymbol{w}_{N}\right) \otimes D_{N, \theta}\left(\boldsymbol{w}_{N}\right)} \rightarrow \overline{\mathbb{A}_{\theta}(\boldsymbol{w}) \otimes \mathbb{A}_{\theta}(\boldsymbol{w})} \quad \text { weakly in } L^{2}\left(0, T ; H^{-\frac{1}{2}+2 \theta}\left(\mathbb{T}_{3}\right)^{3 \times 3}\right) .
$$

Similarly, we get

$$
\begin{array}{ll}
D_{N, \theta}\left(\boldsymbol{w}_{N}\right) \otimes \boldsymbol{B}_{N} \rightarrow \mathbb{A}_{\theta}(\boldsymbol{w}) \otimes \boldsymbol{B} & \text { weakly in } L^{2}\left(0, T ; H^{-\frac{1}{2}}\left(\mathbb{T}_{3}\right)^{3 \times 3}\right), \\
\boldsymbol{B}_{N} \otimes D_{N, \theta}\left(\boldsymbol{w}_{N}\right) \rightarrow \boldsymbol{B} \otimes \mathbb{A}_{\theta}(\boldsymbol{w}) & \text { weakly in } L^{2}\left(0, T ; H^{-\frac{1}{2}}\left(\mathbb{T}_{3}\right)^{3 \times 3}\right) .
\end{array}
$$

These convergence results allow us to prove that $(\boldsymbol{w}, \boldsymbol{B}, q)$ is a distributional solution to the mean MHD equations (5.1). This finishes the proof of Theorem 5.1.

\section{REFERENCES}

[1] H. ALI, On a critical Leray- $\alpha$ model of turbulence, Nonlinear Analysis: Real World Application, 14:3 (2013), pp. 1563-1584.

[2] H. Ali, Large Eddy Simulation for Turbulent Flows with Critical Regularization, Journal of Mathematical Analysis and Applications, 394 (2012), pp. 291-304.

[3] H. ALI, Theory for the rotational deconvolution model with fractional regularization, Applicable Analysis, 2013. Available from: http://www.tandfonline.com/doi/abs/10.1080/00036811.2013.772139

[4] L.C. Berselli, D. Catania, And R. Lewandowski, Convergence of approximate deconvolution models to the mean magnetohydrodynamics equations: Analysis of two models, Journal of Mathematical Analysis and Applications, 401 (2013), pp. 864-880.

[5] L. C. Berselli and R. Lewandowski, Convergence of approximate deconvolution models to the mean Navier-Stokes equations, Annales de l'Institut Henri Poincare (C), NonLinear Analysis, 29 (2012), pp, 171-198.

[6] D. Catania And P. Secchi, Global existence for two regularized MHD models in three spacedimension, Port. Math., 68:1 (2011), pp. 41-52.

[7] J.-Y. Chemin, I. Gallagher, AND P. ZHAng, Sums of large global solutions to the incompressible Navier-Stokes equations, Journal für die reine und angewandte Mathematik (Crelles Journal), 2013.

[8] A. Dunca And Y. Epshteyn, On the Stolz-Adams deconvolution model for the large-eddy simulation of turbulent flows, SIAM J. Math. Anal., 37:6 (2006), pp. 1890-1902.

[9] A. Dunca And R. Lewandowski, Error estimates in approximate deconvolution models, submitted, 2012. Available from: http://arxiv.org/abs/1111.6362

[10] M. Germano, Differential filters for the large eddy simulation of turbulent flows, Phys. Fluids, 29 (1986), pp. $1755-1757$.

[11] A. LARIOS AND E. S. TiTI, On the higher-order global regularity of the inviscid Voigtregularization of three-dimensional hydrodynamic models, Discrete and Continuous Dynamical Systems, 14:2 (2010), pp. 603-627. 
[12] A. LaRios And E. S. Titi, Higher order global regularity of an inviscid Voigt-regularization of the three-dimensional inviscid resistive magnetohydrodynamic equations, J. Math. Fluid Mech., 2013.

[13] J. L. Lions and E. Magenes, Problèmes aux limites non homogènes et applications, Dunod, Paris, 1968.

[14] J. Simon, Compact sets in the spaces $L^{p}(0, t ; b)$, Annali di Mat. Pura ed Applic., 146 (1987), pp. 65-96.

[15] S. Stolz and N. A. Adams, An approximate deconvolution procedure for large-eddy simulation, Phys. Fluids, 11 (1999), pp. 1699-1701.

[16] S. Stolz, N. A. Adams, And L. Kleiser, An approximate deconvolution model for large-eddy simulation with application to incompressible wall bounded flows, Phys. Fluids, 13 (2001), pp. 997-1015.

[17] T. TAO, Global regularity for a logarithmically supercritical hyperdissipative Navier-Stokes equation, Anal. PDE, 2:361 (2009), pp. 361-366.

[18] R. Temam, Navier-Stokes Equations and Nonlinear Functional Analysis, CBMS Regional Conference series, No. 41. SIAM,Philadelphia., 1983.

[19] W. Walter, Differential and integral inequalities, Springer-Verlag, New York, 1970.

[20] J. Wu, Global regularity for a class of generalized magnetohydrodynamic equations, J. Math. Fluid Mech., 13:2 (2011), pp. 295-305. 
H. ALI 